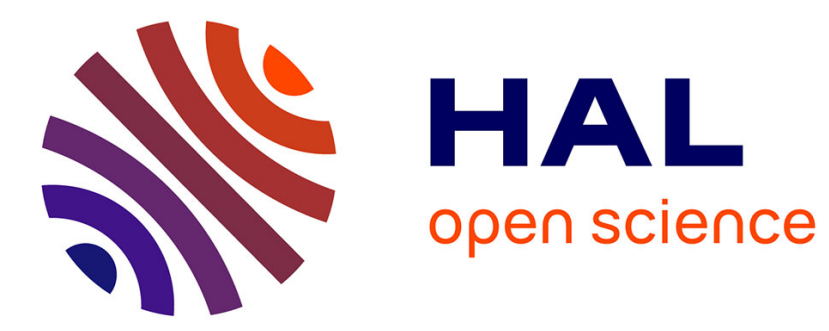

\title{
On the Gross-Pitaevskii equation for trapped dipolar quantum gases
}

\author{
Rémi Carles, Peter Markowich, Christof Sparber
}

\section{To cite this version:}

Rémi Carles, Peter Markowich, Christof Sparber. On the Gross-Pitaevskii equation for trapped dipolar quantum gases. Nonlinearity, 2008, 21, pp.2569-2590. 10.1088/0951-7715/21/11/006 . hal-00277453

\section{HAL Id: hal-00277453 \\ https://hal.science/hal-00277453}

Submitted on 6 May 2008

HAL is a multi-disciplinary open access archive for the deposit and dissemination of scientific research documents, whether they are published or not. The documents may come from teaching and research institutions in France or abroad, or from public or private research centers.
L'archive ouverte pluridisciplinaire HAL, est destinée au dépôt et à la diffusion de documents scientifiques de niveau recherche, publiés ou non, émanant des établissements d'enseignement et de recherche français ou étrangers, des laboratoires publics ou privés. 


\title{
ON THE GROSS-PITAEVSKII EQUATION FOR TRAPPED DIPOLAR QUANTUM GASES
}

\author{
RÉMI CARLES, PETER A. MARKOWICH, AND CHRISTOF SPARBER
}

\begin{abstract}
We study the time-dependent Gross-Pitaevskii equation describing Bose-Einstein condensation of trapped dipolar quantum gases. Existence and uniqueness as well as the possible blow-up of solutions are studied. Moreover, we discuss the problem of dimension-reduction for this nonlinear and nonlocal Schrödinger equation.
\end{abstract}

\section{INTRODUCTION}

The success of atomic Bose-Einstein condensation has stimulated great interest in the properties of trapped quantum gases. Recent developments in the manipulation of such ultra-cold atoms have paved the way towards Bose-Einstein condensation in atomic gases where dipole-dipole interactions between the particles are important. In [21] Yi and You were the first to introduce a pseudo-potential appropriate to describe such systems in which particles interact via short-range repulsive forces and long-range (partly attractive) dipolar forces. Describing the corresponding Bose-Einstein condensates within the realm of the Gross-Pitaevskii (mean field) approximation, one is led to the following nonlinear Schrödinger equation for the macroscopic wave function of the condensate [13, 16, 17, 22, 23]:

$$
i \hbar \partial_{t} \psi+\frac{\hbar^{2}}{2 m} \Delta \psi=V(x) \psi+g|\psi|^{2} \psi+d^{2}\left(K *|\psi|^{2}\right) \psi, \quad x \in \mathbb{R}^{3}, t>0,
$$

where $|g|=4 \pi \hbar^{2} N|a| / m$. Here, we denote by $N$ the total number of particles within the condensate, whereas $m$ denotes the mass of an individual particle and $a$ its corresponding scattering length (which can be experimentally tuned to be either positive or negative). The wave function $\psi$ is then normalized, such that $\|\psi\|_{L^{2}}^{2}=1$. The potential $V(x)$, for $x=\left(x_{1}, x_{2}, x_{3}\right) \in \mathbb{R}^{3}$, describes the electromagnetic trap for the condensate and is usually chosen to be a harmonic confinement, i.e.

$$
V(x)=\frac{m}{2}\left(\omega_{1}^{2} x_{1}^{2}+\omega_{2}^{2} x_{2}^{2}+\omega_{3}^{2} x_{3}^{2}\right) .
$$

The real-valued constants $\omega_{1}, \omega_{2}, \omega_{3}$ then represent the corresponding trap frequency in each spatial direction. Finally, $d$ denotes the dipole moment (in Gaussian units) and

$$
K(x)=\frac{1-3 \cos ^{2} \theta}{|x|^{3}}
$$

2000 Mathematics Subject Classification. 35Q55, 35A05, 81Q99.

Key words and phrases. Bose-Einstein condensates, Gross-Pitaevskii equation, dipole interaction, dimension reduction.

This work has been supported by the KAUST Investigator Award of P. Markowich. R.C. is partially supported by the ANR project SCASEN. P.M. acknowledges support from the Royal Society through his "Wolfson Research Merit Award". C. S. has been supported by the "APART grant" of the Austrian Academy of Science. 
where $\theta$ stands for the angle between $x \in \mathbb{R}^{3}$ and the dipole axis $n \in \mathbb{R}^{3}$, with $|n|=1$. In other words $\theta$ is defined via

$$
\cos \theta=\frac{x \cdot n}{|x|}
$$

To our knowledge a rigorous mathematical study of (1.1) has not been given so far. Note that the interaction kernel (1.3) is indeed highly singular and it is therefore not clear a priori if the corresponding convolution operator is well defined. In particular, not even the existence and uniqueness of solutions to (1.1) has been established yet, and it will be one of the main tasks of this work to do so. Moreover, we shall be interested in the mathematical problem of dimension reduction. Quasi two-dimensional (pancake shaped) or even quasi one-dimensional (cigar shaped) Bose-Einstein condensates can be obtained experimentally by appropriately tuning the trap-frequencies $\omega_{1}, \omega_{2}, \omega_{3}$. The mathematical derivation of effective models in lower dimensions via the corresponding scaling limits is therefore of great practical importance.

For the mathematical analysis it is more convenient to rescale (1.1) in dimensionless form (see e.g. [3]), to arrive at the following model

$$
i \partial_{t} \psi+\frac{1}{2} \Delta \psi=V(x) \psi+\lambda_{1}|\psi|^{2} \psi+\lambda_{2}\left(K *|\psi|^{2}\right) \psi, \quad x \in \mathbb{R}^{3},
$$

where $\lambda_{1}=4 \pi a N / a_{0}$ and $\lambda_{2}=d^{2} /\left(\hbar \omega_{0} a_{0}^{3}\right)$. Here, we denote by $a_{0}=\sqrt{\hbar / m \omega_{0}}$ the ground state length of a harmonic oscillator corresponding to $\omega_{0}=\frac{1}{3}\left(\omega_{1}+\omega_{2}+\omega_{3}\right)$. In the upcoming analysis $\lambda_{1}, \lambda_{2}$ will simply be assumed to be two given, real-valued parameters.

The paper is then organized as follows: We start by collecting several properties of the interaction kernel $K$ in Section 2. After that, we set up a local in time existence theory in Section 3, before proving several different global existence results in Section 4. Section 5 is devoted to the question of finite time blow-up of solutions and also includes an additional global in time existence result for rather particular circumstances. Finally, we shall study the dimensional reduction of our model in Section 6.

\section{Some PROPERTIES OF THE DIPOLE KERNEL}

To simplify notations, we shall from now on assume, without restriction of generality, that $n=(0,0,1)$. The dipole-interaction kernel $K$ then reads:

$$
K(x)=\frac{x_{1}^{2}+x_{2}^{2}-2 x_{3}^{2}}{|x|^{5}} .
$$

Even though this kernel is highly singular (like $1 /|x|^{3}$ ), it defines a rather smooth operator. The technical reason for this is that the average of $K$ vanishes on spheres.

Lemma 2.1. The operator $\mathcal{K}: u \mapsto K * u$ can be extended as a continuous operator on $L^{p}\left(\mathbb{R}^{3}\right)$ for all $1<p<\infty$.

Proof. We notice that $\Omega: x \mapsto 1-3 \cos ^{2} \theta=1-3 \frac{x_{3}^{2}}{|x|^{2}}$ is Lipschitzean on $\mathbb{S}^{2}$, homogeneous of degree zero, and

$$
K(x)=\frac{\Omega(x)}{|x|^{3}}
$$

for $x \in \mathbb{R}^{3}$. We also note that the average of $\Omega$ on spheres vanishes:

$$
\int_{\mathbb{S}^{2}} \Omega=0
$$

The lemma then follows from the Calderón-Zygmund Theorem (see e.g. [18]). 
Remark 2.2. The fact that the average of $K$ vanishes on $\mathbb{S}^{2}$ implies that the dipole-nonlinearity vanishes when applied to radially symmetric wave functions $\psi=\psi(|x|)$. In this case (1.4) simplifies to the classical (cubic nonlinear) GrossPitaevskii equation.

The continuity at the $L^{2}$-level can also be seen by computing the Fourier transform of $K$, which turns out to be essentially bounded, i.e. $\widehat{K} \in L^{\infty}\left(\mathbb{R}^{3}\right)$. In the following we shall use the explicit formula for this Fourier transform several times, see also [13, 16].

Lemma 2.3. Define the Fourier transform on the Schwartz space as

$$
\mathcal{F} u(\xi) \equiv \widehat{u}(\xi)=\int_{\mathbb{R}^{3}} e^{-i x \cdot \xi} u(x) d x, \quad u \in \mathcal{S}\left(\mathbb{R}^{3}\right)
$$

Then the Fourier transform of $K$ is given by

$$
\widehat{K}(\xi)=\frac{4 \pi}{3}\left(3 \cos ^{2} \Theta-1\right)=\frac{4 \pi}{3}\left(3 \frac{\xi_{3}^{2}}{|\xi|^{2}}-1\right)=\frac{4 \pi}{3}\left(\frac{2 \xi_{3}^{2}-\xi_{1}^{2}-\xi_{2}^{2}}{|\xi|^{2}}\right)
$$

where $\Theta$ stands for the angle between $\xi$ and the dipole axis $n=(0,0,1)$.

Proof. We use the decomposition of $e^{-i x \cdot \xi}$ into spherical harmonics

$$
e^{-i x \cdot \xi}=\sum_{m=0}^{\infty}(-i)^{m}(2 m+1) j_{m}(|x||\xi|) P_{m}(\cos \phi)
$$

where $j_{m}$ and $P_{m}$ stand for the spherical Bessel function and Legendre polynomial of order $m$, respectively, and $\phi$ denotes the angle between $x$ and $\xi$, i.e. $x \cdot \xi=|x||\xi| \cos \phi$ (see e.g. [1]). Denoting by $\omega$ and $\omega^{\prime}$ the corresponding spherical coordinates of $x$ and $\xi$, respectively, we shall further expand $P_{m}$ via

$$
P_{m}(\cos \phi)=\frac{4 \pi}{2 m+1} \sum_{\ell=-m}^{m} Y_{\ell m}^{*}(\omega) Y_{\ell m}\left(\omega^{\prime}\right)
$$

where $Y_{\ell m}$ are the spherical harmonics. Note that, since

$$
P_{2}(x)=\frac{1}{2}\left(3 x^{2}-1\right)
$$

we have the following identity

$$
K(x)=-2 \frac{P_{2}(\cos \theta)}{|x|^{3}}=-2 \sqrt{\frac{4 \pi}{5}} \frac{Y_{20}(\omega)}{|x|^{3}},
$$

where we recall that $\theta$ is the angle between $x$ and the dipole axis $n$. 
Putting all of this together we can compute the Fourier transform of $K$ via

$$
\begin{aligned}
\widehat{K}(\xi)= & \int_{\mathbb{R}^{3}} \sum_{m=0}^{\infty}(-i)^{m}(2 m+1) j_{m}(|x||\xi|) P_{m}(\cos \phi) K(x) d x \\
= & -2 \sqrt{\frac{4 \pi}{5}} \sum_{m=0}^{\infty}(-i)^{m}(2 m+1) \int_{0}^{\infty} j_{m}(r|\xi|) \frac{1}{r^{3}} r^{2} d r \times \\
& \int_{\omega \in \mathbb{S}^{2}} P_{m}(\cos \phi) Y_{20}(\omega) d S(\omega) \\
= & -2 \sqrt{\frac{4 \pi}{5}} \sum_{m=0}^{\infty}(-i)^{m}(2 m+1) \int_{0}^{\infty} j_{m}(r|\xi|) \frac{d r}{r} \times \\
& \frac{4 \pi}{2 m+1} \int_{\omega \in \mathbb{S}^{2}} \sum_{\ell=-m}^{m} Y_{\ell m}^{*}(\omega) Y_{\ell m}\left(\omega^{\prime}\right) Y_{20}(\omega) d S(\omega) \\
= & -2 \sqrt{\frac{4 \pi}{5}}(-i)^{2} \times 5 \int_{0}^{\infty} j_{2}(r|\xi|) \frac{d r}{r} \times \frac{4 \pi}{5} Y_{20}\left(\omega^{\prime}\right),
\end{aligned}
$$

where in the last equality we have used the fact that the $\left\{Y_{\ell m}\right\}$ form an orthonormal basis of $L^{2}\left(\mathbb{S}^{2}\right)$. We thus have, for $\xi \neq 0$,

$$
\begin{aligned}
\widehat{K}(\xi) & =8 \pi \sqrt{\frac{4 \pi}{5}} Y_{20}\left(\omega^{\prime}\right) \int_{0}^{\infty} j_{2}(r|\xi|) \frac{d r}{r}=8 \pi P_{2}(\cos \Theta) \int_{0}^{\infty} j_{2}(r) \frac{d r}{r} \\
& =4 \pi\left(3 \cos ^{2} \Theta-1\right) \int_{0}^{\infty} j_{2}(r) \frac{d r}{r} .
\end{aligned}
$$

It remains to compute the radial integral in this formula. In view of the identity

$$
\frac{d}{d r}\left(\frac{j_{1}(r)}{r}\right)=-\frac{j_{2}(r)}{r}
$$

we have

Recalling that

$$
\int_{0}^{\infty} j_{2}(r) \frac{d r}{r}=\lim _{R \rightarrow 0} \int_{R}^{\infty} j_{2}(r) \frac{d r}{r}=\lim _{R \rightarrow 0} \frac{j_{1}(R)}{R}
$$

$$
j_{1}(R)=\frac{\sin R}{R^{2}}-\frac{\cos R}{R},
$$

we thus find $\int_{0}^{\infty} j_{2}(r) / r d r=1 / 3$, which finishes the proof.

\section{LOCAL IN TIME EXISTENCE}

In view of the forthcoming dimension reduction analysis in Section 6, we allow the spatial dimension to be smaller than three and consider, instead of (1.4), the following initial value problem

$$
\left\{\begin{aligned}
i \partial_{t} \psi+\frac{1}{2} \Delta \psi & =V_{d}(x) \psi+\lambda_{1}|\psi|^{2} \psi+\lambda_{2}\left(K_{d} *|\psi|^{2}\right) \psi, \quad x \in \mathbb{R}^{d}, \\
\left.\psi\right|_{t=0} & =\varphi(x)
\end{aligned}\right.
$$

where $1 \leqslant d \leqslant 3$. We assume $\lambda_{1}, \lambda_{2} \in \mathbb{R}$ and the potential $V_{d}$ to be quadratic in $d$ dimensions, i.e.

$$
V_{d}(x)=\frac{1}{2} \sum_{j=1}^{d} \omega_{j}^{2} x_{j}^{2}, \quad \omega_{j} \in \mathbb{R} .
$$

The interaction kernel $K_{d}$ will not be specified in detail (except that $K_{3} \equiv K$ ). Rather, we shall only assume that the operator $\mathcal{K}_{d}: u \mapsto K_{d} * u$ is bounded on $L^{2}\left(\mathbb{R}^{d}\right)$, a property already known to be satisfied in $d=3$, see Lemma 2.1. In 
$d=1,2$ we shall check that this holds true after we succeeded in deriving the precise expressions of $K_{d}$ via dimension reduction.

The following two important physical quantities are formally conserved by the time-evolution associated to (3.1):

$$
\begin{aligned}
\text { Mass: } M= & \|\psi(t)\|_{L^{2}}^{2} . \\
\text { Energy: } E= & \frac{1}{2}\|\nabla \psi(t)\|_{L^{2}}^{2}+\int_{\mathbb{R}^{d}} V_{d}(x)|\psi(t, x)|^{2} d x+\frac{\lambda_{1}}{2}\|\psi(t)\|_{L^{4}}^{4} \\
& +\frac{\lambda_{2}}{2} \int_{\mathbb{R}^{d}}\left(K_{d} *|\psi|^{2}\right)(t, x)|\psi(t, x)|^{2} d x .
\end{aligned}
$$

Note that for the conservation of energy (which can be derived formally by multiplying (3.1) by $\partial_{t} \bar{\psi}$, taking real parts and integrating in $x$ ), we use the fact that $K$ is even. These two quantities naturally lead to the introduction of an energy space associated with the linear case $\lambda_{1}=\lambda_{2}=0$ :

$$
\Sigma=\left\{u \in L^{2}\left(\mathbb{R}^{d}\right) ; \quad\|u\|_{\Sigma}^{2}:=\|u\|_{L^{2}}^{2}+\|\nabla u\|_{L^{2}}^{2}+\|x u\|_{L^{2}}^{2}<\infty\right\} .
$$

Let us ignore the dipole nonlinearity for the moment: $\lambda_{2}=0 . \Sigma$ is a natural space to study (3.1), where several results concerning the Cauchy problem are available (see e.g. [9]). Moreover, it is known that working only in $L^{2}\left(\mathbb{R}^{3}\right)$ is not sufficient to prove (local) well-posedness; see e.g. [7] and references therein. Also note that the Cauchy problem is ill-posed in $H^{1}\left(\mathbb{R}^{d}\right)$, due to the rotation of phase space induced by the harmonic oscillator (see [8] for both linear and nonlinear cases). However, including the dipole-nonlinearity in this setting is not completely straightforward as we can not allow for any (weak) derivative to be put on $K_{d}$, since the corresponding convolution operator is no longer well defined. The only possible way to circumvent this problem seems to be the use of Strichartz estimates. To this end, we shall introduce in the following subsection the main technical tools needed later on in the local existence proof.

3.1. Technical preliminaries. Let $1 \leqslant d \leqslant 3$, and $V_{d}$ be quadratic as in (3.2). Introduce the group

$$
U(t)=e^{-i t H}, \quad \text { where } H=-\frac{1}{2} \Delta+V_{d},
$$

which generates the time-evolution for the linear problem. We first remark that in view of Mehler's formula (see e.g. [10]), the group $U(\cdot)$ is not only bounded on $L^{2}\left(\mathbb{R}^{d}\right)$, but also enjoys dispersive properties for small time. More precisely it holds

$$
\|U(t) \varphi\|_{L^{\infty}\left(\mathbb{R}^{d}\right)} \leqslant \frac{C}{|t|^{d / 2}}\|\varphi\|_{L^{1}\left(\mathbb{R}^{d}\right)}, \quad \text { for }|t| \leqslant \delta,
$$

for some $\delta>0$. Note that for an harmonic potential as in (3.2), $\delta$ is necessarily finite, since $H$ has eigenvalues.

Remark 3.1. This dispersive estimate (3.5) turns out to be valid for external potentials which are not exactly quadratic (nor even positive): it suffices to have $V_{d} \in C^{\infty}\left(\mathbb{R}^{d} ; \mathbb{R}\right)$ with $\partial^{\alpha} V_{d} \in L^{\infty}\left(\mathbb{R}^{d}\right)$ as soon as $|\alpha| \geqslant 2$ for the above estimate to remain valid. In that case, the proofs we present below remain valid, see e.g. [6] and references therein.

An important consequence of the dispersive estimate (3.5), and of the fact that $U(\cdot)$ is unitary on $L^{2}$, is the existence of Strichartz estimates, which require the following definition of admissible index pairs. 
Definition 3.2. A pair $(q, r)$ is admissible if $2 \leqslant r \leqslant \frac{2 d}{d-2}$ (resp. $2 \leqslant r \leqslant \infty$ if $d=1,2 \leqslant r<\infty$ if $d=2$ ) and

$$
\frac{2}{q}=\delta(r):=d\left(\frac{1}{2}-\frac{1}{r}\right) .
$$

Following [11, 20, 15], we have:

Lemma 3.3 (Strichartz estimates). Let $(q, r),\left(q_{1}, r_{1}\right)$ and $\left(q_{2}, r_{2}\right)$ be admissible pairs. Let $I$ be some finite time interval containing the origin, $0 \in I$.

1. There exists $C_{r}=C(r, I)$, such that for any $\varphi \in L^{2}\left(\mathbb{R}^{d}\right)$ it holds

$$
\|U(\cdot) \varphi\|_{L^{q}\left(I ; L^{r}\right)} \leqslant C_{r}\|\varphi\|_{L^{2}} .
$$

2. There exists $C_{r_{1}, r_{2}}=C\left(r_{1}, r_{2}, I\right)$, such that for any $F \in L^{q_{2}^{\prime}}\left(I ; L^{r_{2}^{\prime}}\right)$ it holds

$$
\left\|\int_{I \cap\{s \leq t\}} U(t-s) F(s) d s\right\|_{L^{q_{1}\left(I ; L^{r_{1}}\right)}} \leqslant C_{r_{1}, r_{2}}\|F\|_{L^{q_{2}^{\prime}\left(I ; L^{r_{2}^{\prime}}\right)}} .
$$

As underscored above, taking for $\varphi$ an eigenfunction of the (anisotropic) harmonic oscillator shows that in general the constants $C_{r}$ and $C_{r_{1}, r_{2}}$ do depend on the length of the time interval $I$, unless all the $\omega_{j}$ 's in (3.2) are zero.

In the following we shall use two special admissible index pairs to apply the above lemma, namely

$$
(q, r)=(\infty, 2) \text { and }(q, r)=\left(\frac{8}{d}, 4\right)
$$

The idea for the proofs of local in time existence and uniqueness is then to apply a fixed point argument on the Duhamel's formula associated to (3.1). This step turns out to follow exactly the same lines as in the proof of local existence without dipole, $\lambda_{2}=0$. To this end Strichartz estimates based on (3.8) will be invoked several times.

3.2. Constructing a local solution in the energy space. With the above technical tools in hand we can now state the first main result of this work.

Proposition 3.4. Let $1 \leqslant d \leqslant 3, V_{d}$ be quadratic, $\lambda_{1}, \lambda_{2} \in \mathbb{R}$, and $\varphi \in \Sigma$. Assume that the operator $\mathcal{K}_{d}: u \mapsto K_{d} * u$ is bounded on $L^{2}\left(\mathbb{R}^{d}\right)$. Then there exists $T$, depending only on (upper bounds for) $\|\varphi\|_{\Sigma}$, such that (3.1) has a unique solution $\psi \in X_{T}$, where

$$
X_{T}=\left\{\psi \in C([0, T] ; \Sigma) ; \psi, \nabla \psi, x \psi \in C\left([0, T] ; L^{2}\left(\mathbb{R}^{d}\right)\right) \cap L^{8 / d}\left([0, T] ; L^{4}\left(\mathbb{R}^{d}\right)\right)\right\} .
$$

Moreover, the mass $M$ and the energy E, defined in (3.3), (3.4), are conserved for $t \in[0, T]$.

The space $L^{8 / d}\left([0, T] ; L^{4}\left(\mathbb{R}^{d}\right)\right)$ is here to ensure uniqueness. The appearance of this space will become clear during the course of the proof. The main technical remark is that since $\mathcal{K}_{d}$ is continuous on $L^{2}\left(\mathbb{R}^{d}\right)$ by assumption, the nonlocal term in (3.1) can be estimated like the local cubic nonlinearity.

Proof. The proof of the conservations of mass and energy is omitted here. We refer to [9] for a general argument, easy to adapt to the present case. Now, consider Duhamel's formula associated to (3.1)

$$
\psi(t)=U(t) \varphi-i \lambda_{1} \int_{0}^{t} U(t-s)\left(|\psi|^{2} \psi\right)(s) d s-i \lambda_{2} \int_{0}^{t} U(t-s)\left(\left(K_{d} *|\psi|^{2}\right) \psi\right)(s) d s
$$


and denote $\Phi(\psi)(t)=U(t) \varphi+S_{1}(t)+S_{2}(t)$, with

$$
\begin{aligned}
& S_{1}(t)=-i \lambda_{1} \int_{0}^{t} U(t-s)\left(|\psi|^{2} \psi\right)(s) d s, \\
& S_{2}(t)=-i \lambda_{2} \int_{0}^{t} U(t-s)\left(\left(K_{d} *|\psi|^{2}\right) \psi\right)(s) d s .
\end{aligned}
$$

Since at this stage, we are interested in a local result only, we may assume that $T \in] 0,1]$. For $\varphi \in \Sigma$, let $R=\|\varphi\|_{\Sigma}$, and introduce

$$
\begin{aligned}
X_{T}(R)=\left\{\psi \in X_{T} ;\|A \psi\|_{L^{\infty}\left([0, T] ; L^{2}\right)} \leqslant 4 C_{2} R,\right. & \\
& \text { and } \left.\|A \psi\|_{L^{8 / d}\left([0, T] ; L^{4}\right)} \leqslant 4 C_{4} R, \quad \forall A \in\{\mathrm{Id}, \nabla, x\}\right\} .
\end{aligned}
$$

The constants $C_{2}$ and $C_{4}$ are those which appear in the first point of Lemma 3.3, with $I=[0,1]$. Apart from them we shall in the following denote all the irrelevant constants by $C$, whose value may therefore change from one line to another.

To prove Proposition 3.4, we first show that for $T \in] 0,1]$ sufficiently small, $\Phi$ leaves $X_{T}(R)$ invariant. Then, up to demanding $T$ to be even smaller, we show that $\Phi$ is a contraction on $X_{T}(R)$, for the topology of $L^{8 / d}\left([0, T] ; L^{4}\right)$. As remarked in [14], $X_{T}(R)$, equipped with this topology, is complete, and thus Proposition 3.4 follows, since $T$ will only depend on $d$ and $R$.

Step 1 (stability): Let $\psi \in X_{T}(R)$ and denote $L_{T}^{q} L^{r}=L^{q}\left([0, T] ; L^{r}\left(\mathbb{R}^{d}\right)\right)$. The Strichartz estimates yield:

$$
\begin{aligned}
\|\Phi(\psi)\|_{L_{T}^{\infty} L^{2}} & \leqslant\|U(\cdot) \varphi\|_{L_{T}^{\infty} L^{2}}+\left\|S_{1}\right\|_{L_{T}^{\infty} L^{2}}+\left\|S_{2}\right\|_{L_{T}^{\infty} L^{2}} \\
& \leqslant C_{2} R+C_{2,4}\left\||\psi|^{2} \psi\right\|_{L_{T}^{8 /(8-d)} L^{4 / 3}}+C_{2,4}\left\|\left(K_{d} *|\psi|^{2}\right) \psi\right\|_{L_{T}^{8 /(8-d)} L^{4 / 3}} .
\end{aligned}
$$

Similarly, it holds

$$
\|\Phi(\psi)\|_{L_{T}^{8 / d} L^{4}} \leqslant C_{4} R+C_{4,4}\left\||\psi|^{2} \psi\right\|_{L_{T}^{8 /(8-d)} L^{4 / 3}}+C_{4,4}\left\|\left(K_{d} *|\psi|^{2}\right) \psi\right\|_{L_{T}^{8 /(8-d)} L^{4 / 3}} .
$$

We shall only work on the latter estimate as the $L_{T}^{\infty} L^{2}$ estimate can handled exactly in the same way. Denote $(q, r)=(8 / d, 4)$ and remark the following identities

$$
\frac{3}{4}=\frac{1}{r^{\prime}}=\frac{3}{r} \quad ; \quad \frac{8-d}{8}=\frac{1}{q^{\prime}}=\frac{1}{q}+\frac{2}{k}, \text { with } k=\frac{8}{4-d} .
$$

Hölder's inequality thus yields

$$
\left\||\psi|^{2} \psi\right\|_{L_{T}^{8 /(8-d)} L^{4 / 3}} \leqslant\|\psi\|_{L_{T}^{k} L^{r}}^{2}\|\psi\|_{L_{T}^{q} L^{r}}
$$

By assumption, the operator $\mathcal{K}_{d}: u \mapsto K_{d} *|u|^{2}$ is bounded on $L^{2}\left(\mathbb{R}^{d}\right)$ and so we have (recall that $r=4$ ):

$$
\begin{aligned}
\left\|\left(K_{d} *|\psi|^{2}\right) \psi\right\|_{L_{T}^{8 /(8-d)} L^{4 / 3}} & \leqslant\left\|K_{d} *|\psi|^{2}\right\|_{L_{T}^{k / 2} L^{r / 2}}\|\psi\|_{L_{T}^{q} L^{r}} \\
& \leqslant C\left\||\psi|^{2}\right\|_{L_{T}^{k / 2} L^{r / 2}}\|\psi\|_{L_{T}^{q} L^{r}} \leqslant C\|\psi\|_{L_{T}^{k} L^{r}}^{2}\|\psi\|_{L_{T}^{q} L^{r}} .
\end{aligned}
$$

We note that this is exactly the same estimate as for the local cubic term, up to the norm of $\mathcal{K}_{d}$. From Sobolev embedding (in space), we get

$$
\|\psi\|_{L_{T}^{k} L^{r}} \leqslant C\|\psi\|_{L_{T}^{k} H^{1}} \leqslant C T^{1 / k}\|\psi\|_{L_{T}^{\infty} H^{1}} \leqslant C T^{1 / k} C_{2} R .
$$

Therefore,

$$
\|\Phi(\psi)\|_{L_{T}^{q} L^{r}} \leqslant C_{4} R+C T^{2 / k} R^{3},
$$

and if $T \in] 0,1]$ is sufficiently small, $\|\Phi(\psi)\|_{L_{T}^{q} L^{r}} \leqslant 4 C_{4} R$.

Next, to estimate $\nabla \Phi(\psi)$, we denote by $[A, B]=A B-B A$ the usual commutator and compute

$$
[\nabla, H]=[\nabla, V]=\nabla V, \quad[x, H]=\frac{1}{2}[x, \Delta]=-\nabla .
$$


By assumption, $|\nabla V(x)| \leqslant C|x|$, which shows that we will indeed get a closed family of estimates for $\nabla \Phi(\psi)$ and $x \Phi(\psi)$. For instance,

$$
\begin{aligned}
\nabla \Phi(\psi)(t)= & U(t) \nabla \varphi-i \lambda_{1} \int_{0}^{t} U(t-s) \nabla\left(|\psi|^{2} \psi\right)(s) d s \\
& -i \lambda_{2} \int_{0}^{t} U(t-s) \nabla\left(\left(K_{d} *|\psi|^{2}\right) \psi\right)(s) d s \\
& -i \int_{0}^{t} U(t-s)(\Phi(\psi(s)) \nabla V) d s .
\end{aligned}
$$

From the Strichartz estimates, we thus have

$$
\|U(\cdot) \nabla \varphi\|_{L_{T}^{\infty} L^{2}} \leqslant C_{2} R,
$$

and for the local cubic nonlinearity we get

$$
\begin{aligned}
\left\|\int_{0}^{t} U(t-s) \nabla\left(|\psi|^{2} \psi\right)(s) d s\right\|_{L_{T}^{\infty} L^{2}} & \leqslant C_{2,4}\left\|\nabla\left(|\psi|^{2} \psi\right)\right\|_{L_{T}^{q^{\prime} L^{r^{\prime}}}} \leqslant C\left\||\psi|^{2}|\nabla \psi|\right\|_{L_{T}^{q^{\prime} L^{r^{\prime}}}} \\
& \leqslant C\|\psi\|_{L_{T}^{k} L^{r}}^{2}\|\nabla \psi\|_{L_{T}^{q} L^{r}} \leqslant C R\|\psi\|_{L_{T}^{k} L^{r}}^{2} \\
& \leqslant C T^{2 / k} R^{3},
\end{aligned}
$$

where we have used the same computations as above, on $\Phi(\psi)$. For the dipolenonlinearity, we similarly obtain

$$
\begin{aligned}
& \left\|\int_{0}^{t} U(t-s) \nabla\left(\left(K_{d} *|\psi|^{2}\right) \psi\right)(s) d s\right\|_{L_{T}^{\infty} L^{2}} \leqslant C_{2,4}\left\|\nabla\left(\left(K_{d} *|\psi|^{2}\right) \psi\right)\right\|_{L_{T}^{q^{\prime} L^{r^{\prime}}}} \\
& \quad \leqslant C\left\|\left(K_{d} *|\psi|^{2}\right) \nabla \psi\right\|_{L_{T}^{q^{\prime}} L^{r^{\prime}}}+C\left\|\left(K_{d} * \nabla|\psi|^{2}\right) \psi\right\|_{L_{T}^{q^{\prime} L^{r^{\prime}}}} \\
& \quad \leqslant C\left\|K_{d} *|\psi|^{2}\right\|_{L_{T}^{k / 2} L^{r / 2}}\|\nabla \psi\|_{L_{T}^{q} L^{r}}+C\left\|K_{d} * \nabla|\psi|^{2}\right\|_{L_{T}^{(1 / q+1 / k)}{ }_{L^{r / 2}}\|\psi\|_{L_{T}^{k} L^{r}}}\|\psi\|_{L_{T}^{k} L^{r}} \\
& \quad \leqslant C\left\||\psi|^{2}\right\|_{L_{T}^{k / 2} L^{r / 2}}\|\nabla \psi\|_{L_{T}^{q} L^{r}}+C\left\|\nabla|\psi|^{2}\right\|_{L_{T}^{(1 / q+1 / k)^{-1}} L_{L^{r / 2}} \|}\|C\| \psi\left\|_{L_{T}^{k} L^{r}}^{2}\right\| \nabla \psi\left\|_{L_{T}^{q} L^{r}}+C\right\| \psi \nabla \psi \|_{L_{T}^{(1 / q+1 / k)^{-1}} L_{L^{r / 2}}\|\psi\|_{L_{T}^{k} L^{r}}} \\
& \quad \leqslant C\|\psi\|_{L_{T}^{k} L^{r}}^{2}\|\nabla \psi\|_{L_{T}^{q} L^{r}} \leqslant C R\|\psi\|_{L_{T}^{k} L^{r}}^{2} \leqslant C T^{2 / k} R^{3} .
\end{aligned}
$$

This is the same type of estimate as for the local cubic term. Finally, the last term in (3.10) is estimated by

$$
\left\|\int_{0}^{t} U(t-s)(\Phi(\psi(s)) \nabla V) d s\right\|_{L_{T}^{\infty} L^{2}} \leqslant C_{2,2}\|\Phi(\psi) \nabla V\|_{L_{T}^{1} L^{2}} \leqslant C T\|x \Phi(\psi)\|_{L_{T}^{\infty} L^{2}} .
$$

All in all, we find:

$$
\|\nabla \Phi(\psi)\|_{L_{T}^{\infty} L^{2}} \leqslant C_{2} R+C T^{2 / k} R^{3}+C T\|x \Phi(\psi)\|_{L_{T}^{\infty} L^{2}} .
$$

Analogously we obtain (with slightly shorter computations)

$$
\|x \Phi(\psi)\|_{L_{T}^{\infty} L^{2}} \leqslant C_{2} R+C T^{2 / k} R^{3}+C T\|\nabla \Phi(\psi)\|_{L_{T}^{\infty} L^{2}} .
$$

Summing up these two inequalities and taking $T \in] 0,1]$ sufficiently small, we arrive at

$$
\|\nabla \Phi(\psi)\|_{L_{T}^{\infty} L^{2}}+\|x \Phi(\psi)\|_{L_{T}^{\infty} L^{2}} \leqslant 4 C_{2} R
$$

and using the Strichartz estimates again, we also have

$$
\begin{gathered}
\|\nabla \Phi(\psi)\|_{L_{T}^{q} L^{r}} \leqslant C_{4} R+C T^{2 / k} R^{3}+C T\|x \Phi(\psi)\|_{L_{T}^{\infty} L^{2}}, \\
\|x \Phi(\psi)\|_{L_{T}^{q} L^{r}} \leqslant C_{4} R+C T^{2 / k} R^{3}+C T\|\nabla \Phi(\psi)\|_{L_{T}^{\infty} L^{2} .}
\end{gathered}
$$

Up to taking $T$ even smaller, we therefore see that $\Phi$ leaves $X_{T}(R)$ stable. 
Step 2 (contraction): For $\psi_{1}$ and $\psi_{2}$ in $X_{T}(R)$, we have, from Strichartz estimates and the above computations,

$$
\begin{aligned}
\| & \Phi\left(\psi_{1}\right)-\Phi\left(\psi_{2}\right) \|_{L_{T}^{q} L^{r}} \\
\leqslant & C_{4,4}\left\|\left|\psi_{1}\right|^{2} \psi_{1}-\left|\psi_{2}\right|^{2} \psi_{2}\right\|_{L_{T}^{q^{\prime}} L^{r^{\prime}}}+C_{4,4}\left\|\left(K_{d} *\left|\psi_{1}\right|^{2}\right) \psi_{1}-\left(K_{d} *\left|\psi_{2}\right|^{2}\right) \psi_{2}\right\|_{L_{T}^{q^{\prime}} L^{r^{\prime}}} \\
\leqslant & C\left\|\left(\left|\psi_{1}\right|^{2}+\left|\psi_{2}\right|^{2}\right)\left|\psi_{1}-\psi_{2}\right|\right\|_{L_{T}^{q^{\prime}} L^{r^{\prime}}}+C\left\|\left(K_{d} *\left(\left|\psi_{1}\right|^{2}-\left|\psi_{2}\right|^{2}\right)\right) \psi_{1}\right\|_{L_{T}^{q^{\prime}} L^{r^{\prime}}} \\
& +C\left\|\left(K_{d} *\left|\psi_{2}\right|^{2}\right)\left(\psi_{1}-\psi_{2}\right)\right\|_{L_{T}^{q^{\prime}} L^{r^{\prime}}} \\
\leqslant & C T^{2 / k} R^{2}\left\|\psi_{1}-\psi_{2}\right\|_{L_{T}^{q} L^{r}}+C\left\|\left|\psi_{1}\right|^{2}-\left|\psi_{2}\right|^{2}\right\|_{L_{T}^{(1 / q+1 / k)}-1}\left\|\psi_{1}\right\|_{L_{T}^{k} L^{r}} \\
& +C T^{2 / k} R^{2}\left\|\psi_{1}-\psi_{2}\right\|_{L_{T}^{q} L^{r}} \\
\leqslant & C T^{2 / k} R^{2}\left\|\psi_{1}-\psi_{2}\right\|_{L_{T}^{q} L^{r}}
\end{aligned}
$$

Therefore, $\Phi$ is a contraction provided that $T$ is sufficiently small, and hence the result follows.

\section{Global in time existence Results}

Having established the existence and uniqueness of solutions to (3.1) locally in time, we now turn our attention to global in time results. To this end we first note that the only obstruction for global existence in $\Sigma$ is the possible unboundedness of $\nabla \psi$ in $L^{2}\left(\mathbb{R}^{d}\right)$. Indeed, in $\mathbb{R}^{d}$ with $1 \leqslant d \leqslant 3$, the Gagliardo-Nirenberg inequality (see e.g. [9]) yields

$$
\|u\|_{L^{4}}^{4} \leqslant C\|u\|_{L^{2}}^{4-d}\|\nabla u\|_{L^{2}}^{d} .
$$

Thus, the boundedness of $\mathcal{K}_{d}$ on $L^{2}\left(\mathbb{R}^{d}\right)$ together with the Cauchy-Schwarz inequality implies

$$
\begin{aligned}
\left.\left|\int_{\mathbb{R}^{d}}\left(K_{d} *|u|^{2}\right)(x)\right| u(x)\right|^{2} d x \mid & \leqslant\left\|K_{d} *|u|^{2}\right\|_{L^{2}}\left\||u|^{2}\right\|_{L^{2}} \leqslant C\|u\|_{L^{4}}^{4} \\
& \leqslant C\|u\|_{L^{2}}^{4-d}\|\nabla u\|_{L^{2}}^{d},
\end{aligned}
$$

where we have used Gagliardo-Nirenberg inequalities. So we see that in view of the conservation of the mass, the (conserved) energy is the sum of four terms, three of which are bounded provided that $\nabla \psi$ remains bounded in $L^{2}\left(\mathbb{R}^{d}\right)$. Therefore, the fourth term is bounded, that is, $x \psi(t, x)$ is bounded in $L^{2}\left(\mathbb{R}^{d}\right)$. This shows that unless $\|\nabla \psi(t)\|_{L^{2}}$ becomes unbounded, $\|\psi(t)\|_{\Sigma}$ is a continuous function of $t \geqslant 0$. This directly yields the following corollary:

Corollary 4.1. Under the same assumptions as in Proposition 3.4, there exists a $T_{*} \in \mathbb{R}_{+} \cup\{\infty\}$, such that (3.1) has a unique maximal solution in

$$
\left\{\psi \in C \left(\left[0, T_{*}[; \Sigma) ; \psi, \nabla \psi, x \psi \in C\left(\left[0, T_{*}\left[; L^{2}\left(\mathbb{R}^{d}\right)\right) \cap L_{\mathrm{loc}}^{8 / d}\left(\left[0, T_{*}\left[; L^{4}\left(\mathbb{R}^{d}\right)\right)\right\},\right.\right.\right.\right.\right.\right.
$$

such that $\psi_{\mid t=0}=\varphi$. It is maximal in the sense that if $T_{*}<\infty$, then

$$
\|\nabla \psi(t)\|_{L^{2}} \underset{t \rightarrow T_{*}}{\longrightarrow}+\infty .
$$

Having in mind this result, we are now able to study the global in time existence of solutions to (3.1) depending on the spatial dimension. In the following we shall first treat the case $d=1$, where the picture is much more concise, before moving on to the case $d=3$ (the case $d=2$, is similar to the one of three spatial dimensions and thus omitted for simplicity). Note that, in view of the dimensional reduction discussed in Section 6, the one-dimensional case is not purely academic. 
4.1. Global existence for $d=1$. When $d=1$, the solution constructed in the previous section indeed exists for all time. More precisely we have:

Corollary 4.2. Suppose that in Proposition 3.4, $d=1$. Then the solution to (3.1) is global in time, i.e. $T_{*}=\infty$ in Corollary 4.1 .

Proof. This result stems from Gagliardo-Nirenberg inequality, like its classical counterpart when $\lambda_{2}=0$. In view of Corollary 4.1, we just need an a priori estimate for $\|\nabla \psi(t)\|_{L^{2}}$. Indeed the conservation of energy yields

$$
\begin{aligned}
\|\nabla \psi(t)\|_{L^{2}}^{2} & \leqslant 2 E+\left|\lambda_{1}\right|\|\psi(t)\|_{L^{4}}^{4}+\left.\left|\lambda_{2}\right|\left|\int_{\mathbb{R}}\left(K_{1} *|\psi|^{2}\right)(t, x)\right| \psi(t, x)\right|^{2} d x \mid \\
& \leqslant 2 E+C\|\psi(t)\|_{L^{2}}^{3}\|\nabla \psi(t)\|_{L^{2}},
\end{aligned}
$$

where we have again used the estimates (4.1). Since $M=\|\psi(t)\|_{L^{2}}^{2}$ is constant in time, this inequality directly shows that $\|\nabla \psi(t)\|_{L^{2}}$ remains bounded and hence the result is proven.

When $d=1$, a more general setting is possible, which allows us to work with initial data that are only in $L^{2}$ but not necessarily in $\Sigma$ (corresponding to solutions with possibly infinite energy). The following result is an adaptation of the main result in [19].

Theorem 4.3. Let $d=1, V_{1}$ be quadratic, and $\lambda_{1}, \lambda_{2} \in \mathbb{R}$. Assume that the operator $\mathcal{K}_{1}: u \mapsto K_{1} * u$ is bounded on $L^{2}(\mathbb{R})$. Then, for any $\varphi \in L^{2}(\mathbb{R})$, (3.1) has a unique solution

$$
\psi \in C\left(\mathbb{R}_{+} ; L^{2}\right) \cap L_{\text {loc }}^{8}\left(\mathbb{R}_{+} ; L^{4}(\mathbb{R})\right) .
$$

Moreover its total mass $M$ is independent of $t \geqslant 0$.

Proof. We shall first prove that there exists a $T$, depending only on $\|\varphi\|_{L^{2}(\mathbb{R})}$, such that (3.1) has a unique solution

$$
\psi \in C\left([0, T] ; L^{2}\right) \cap L^{8}\left([0, T] ; L^{4}(\mathbb{R})\right),
$$

To this end we resume the same scheme as in the proof of Proposition 3.4 above, but now $R=\|\varphi\|_{L^{2}}$ and

$$
\begin{gathered}
Y_{T}(R)=\left\{\psi \in L^{\infty}\left([0, T] ; L^{2}\right) \cap L^{8}\left([0, T] ; L^{4}\right) ;\|\psi\|_{L^{\infty}\left([0, T] ; L^{2}\right)} \leqslant 2 C_{2} R,\right. \\
\text { and } \left.\|\psi\|_{L^{8}\left([0, T] ; L^{4}\right)} \leqslant 2 C_{4} R\right\} .
\end{gathered}
$$

We simply notice that for $d=1$, the identities (3.9) yield $k=8 / 3$, and so Hölder's inequality in time implies

$$
\|\psi\|_{L_{T}^{k} L^{r}}=\|\psi\|_{L_{T}^{8 / 3} L^{4}} \leqslant T^{1 / 4}\|\psi\|_{L_{T}^{8} L^{4}}=T^{1 / 4}\|\psi\|_{L_{T}^{q} L^{r}}
$$

Following the same lines as in the previous paragraph, we see that $\Phi$ leaves $Y_{T}(R)$ stable, provided that $T$ is sufficiently small. The proof of contraction is the same as in the previous paragraph, up to the modification of the estimate for $\|\psi\|_{L_{T}^{k} L^{r}}$. This yields a local in time existence and uniqueness result and since the existence time depends only on $\|\varphi\|_{L^{2}(\mathbb{R})}$, the conservation of the total mass $M$ directly implies the global in time existence.

In summary the Gross-Pitaevskii equation (3.1) is globally well-posed for $d=1$. The three dimensional case is more involved, though, as we shall see. 
4.2. Global existence for $d=3$ (stable regime). We can now turn to the physically most important case $d=3$.

Theorem 4.4. Under the same assumptions as in Proposition 3.4, suppose that in addition $\lambda_{1} \geqslant \frac{4 \pi}{3} \lambda_{2} \geqslant 0$. Then the solution is global in time, i.e. $T_{*}=\infty$.

In the following, the situation where $\lambda_{1} \geqslant \frac{4 \pi}{3} \lambda_{2} \geqslant 0$ will be called the stable regime (note that $\lambda_{2}>0$ corresponds to the actual physical situation).

Proof. We first note that from Plancherel's formula for $\rho=|\psi|^{2}$ we get

$$
\|\psi(t)\|_{L^{4}}^{4}=\|\rho(t)\|_{L^{2}}^{2}=\frac{1}{(2 \pi)^{3}}\|\widehat{\rho}(t)\|_{L^{2}}^{2} .
$$

Then we simply use the conservation of the energy $E$, as defined in (3.4), to estimate

$$
\begin{aligned}
\|\nabla \psi(t)\|_{L^{2}}^{2}= & 2 E-\int_{\mathbb{R}^{3}} V_{3}(x)|\psi(t, x)|^{2} d x-\lambda_{1}\|\psi(t)\|_{L^{4}}^{4} \\
& -\lambda_{2} \int_{\mathbb{R}^{3}}\left(K_{1} *|\psi|^{2}\right)(t, x)|\psi(t, x)|^{2} d x \\
\leqslant & 2 E-\frac{1}{(2 \pi)^{3}} \int_{\mathbb{R}^{3}}\left(\lambda_{1}+\lambda_{2} \widehat{K}(\xi)\right)|\widehat{\rho}(\xi)|^{2} d \xi,
\end{aligned}
$$

invoking the above given identity. Recalling the explicit formula for $\widehat{K}$ computed in Lemma 2.3, we obtain

$$
\int_{\mathbb{R}^{3}}\left(\lambda_{1}+\lambda_{2} \widehat{K}(\xi)\right)|\widehat{\rho}(\xi)|^{2} d \xi \geqslant \int_{\mathbb{R}^{3}}\left(\lambda_{1}-\frac{4 \pi}{3} \lambda_{2}\right)|\widehat{\rho}(\xi)|^{2} d \xi
$$

By assumption, this quantity is non-negative and hence the a priori estimate

$$
\|\nabla \psi(t)\|_{L^{2}}^{2} \leqslant 2 E
$$

holds true. The result then follows directly from Corollary 4.1.

Having established this result, it is natural to ask what happens in the unstable regime $\lambda_{1}<\frac{4 \pi}{3} \lambda_{2}$. As will shall see, in general we cannot expect a global in time result there, since finite time blow-up of solutions (in the sense of Corollary 4.1) may occur. In particular, it is clear that if blow-up occurs, then the problem of dimension reduction ceases to make sense. The next section is devoted to the study of this problem.

\section{The unstable Regime}

When $d=3$ (or 2), the solution constructed in Proposition 3.4 need not remain in $\Sigma$ for all time. This will be seen from using a general virial computation (see [9]) and following the approach by Zakharov [24] and Glassey [12].

5.1. Finite time blow-up. As a preliminary, we check that the energy $E$ may be negative.

Lemma 5.1. Let $d=3$. Assume that

$$
\lambda_{1}<\frac{4 \pi}{3} \lambda_{2}
$$

There exists $\varphi \in \Sigma$ such that $E<0$, where the energy $E$ is as in Proposition 3.4.

Proof. We first rewrite the last term in the energy, thanks to Plancherel formula:

$$
\int_{\mathbb{R}^{3}}\left(K *|\varphi|^{2}\right)(x)|\varphi(x)|^{2} d x=\frac{1}{(2 \pi)^{3}} \int_{\mathbb{R}^{3}} \widehat{K}(\xi)|\widehat{\rho}(\xi)|^{2} d \xi,
$$


where we have denoted $\rho=|\varphi|^{2}$. In view of Lemma 2.3, we infer

$$
\int_{\mathbb{R}^{3}}\left(K *|\varphi|^{2}\right)(x)|\varphi(x)|^{2} d x=\frac{1}{(2 \pi)^{2}} \int_{\mathbb{R}^{3}}\left(3 \frac{\xi_{3}^{2}}{|\xi|^{2}}-1\right)|\widehat{\rho}(\xi)|^{2} d \xi .
$$

The idea of the proof then consists in choosing $\varphi$ so that $\widehat{\rho}$ has little mass on $\left\{\xi_{3}=0\right\}=\{\xi \cdot n=0\}$. Indeed, using Plancherel formula again, we can rewrite the energy as

$$
\begin{aligned}
E= & \frac{1}{2}\|\nabla \varphi\|_{L^{2}}^{2}+\int_{\mathbb{R}^{3}} V(x)|\varphi(x)|^{2} d x \\
& +\frac{1}{2(2 \pi)^{3}} \int_{\mathbb{R}^{3}}\left(\lambda_{1}+\frac{4 \pi}{3} \lambda_{2}\left(3 \frac{\xi_{3}^{2}}{|\xi|^{2}}-1\right)\right)|\widehat{\rho}(\xi)|^{2} d \xi .
\end{aligned}
$$

Introduce a parameter $\varepsilon>0$, and fix some functions $f \in \mathcal{S}\left(\mathbb{R}^{2}\right)$ and $g \in \mathcal{S}(\mathbb{R})$ independent of $\varepsilon$. Set

$$
\varphi(x)=\varepsilon^{\alpha / 2} f\left(x_{1}, x_{2}\right) g\left(\varepsilon x_{3}\right),
$$

for some constant $\alpha$ to be fixed later. We have

$$
\rho(x)=|\varphi(x)|^{2}=\varepsilon^{\alpha}\left|f\left(x_{1}, x_{2}\right)\right|^{2}\left|g\left(\varepsilon x_{3}\right)\right|^{2}, \quad \widehat{\rho}(\xi)=\varepsilon^{\alpha-1} F\left(\xi_{1}, \xi_{2}\right) G\left(\frac{\xi_{3}}{\varepsilon}\right),
$$

where $F$ and $G$ denote the Fourier transforms of $|f|^{2}$ and $|g|^{2}$, in $\mathcal{S}\left(\mathbb{R}^{2}\right)$ and $\mathcal{S}(\mathbb{R})$, respectively. We now measure the order of magnitude, as $\varepsilon \rightarrow 0$, of each term in the energy:

- Kinetic energy: the leading order term corresponds to the differentiation with respect to $x_{1}$ or $x_{2}$.

$$
\|\nabla \varphi\|_{L^{2}}^{2} \approx \varepsilon^{\alpha} \int_{\mathbb{R}^{3}}\left|\nabla f\left(x_{1}, x_{2}\right)\right|^{2}\left|g\left(\varepsilon x_{3}\right)\right|^{2} d x \approx \varepsilon^{\alpha-1} .
$$

- Potential energy: the leading order term corresponds to the $x_{3}$ component.

- Cubic nonlinear term:

$$
\int_{\mathbb{R}^{3}} V(x)|\varphi(x)|^{2} d x \approx \varepsilon^{\alpha} \int_{\mathbb{R}^{3}} x_{3}^{2}\left|f\left(x_{1}, x_{2}\right)\right|^{2}\left|g\left(\varepsilon x_{3}\right)\right|^{2} d x \approx \varepsilon^{\alpha-3} .
$$

$$
\int|\widehat{\rho}(\xi)|^{2} d \xi=\varepsilon^{2 \alpha-2} \int\left|F\left(\xi_{1}, \xi_{2}\right)\right|^{2}\left|G\left(\frac{\xi_{3}}{\varepsilon}\right)\right|^{2} d \xi \approx \varepsilon^{2 \alpha-1} .
$$

- Finally we compute:

$$
\begin{aligned}
\int \frac{\xi_{3}^{2}}{|\xi|^{2}}|\widehat{\rho}(\xi)|^{2} d \xi & =\varepsilon^{2 \alpha-2} \int \frac{\xi_{3}^{2}}{\xi_{1}^{2}+\xi_{2}^{2}+\xi_{3}^{2}}\left|F\left(\xi_{1}, \xi_{2}\right)\right|^{2}\left|G\left(\frac{\xi_{3}}{\varepsilon}\right)\right|^{2} d \xi \\
& =\varepsilon^{2 \alpha-1} \int \frac{\varepsilon^{2} \xi_{3}^{2}}{\xi_{1}^{2}+\xi_{2}^{2}+\varepsilon^{2} \xi_{3}^{2}}\left|F\left(\xi_{1}, \xi_{2}\right)\right|^{2}\left|G\left(\xi_{3}\right)\right|^{2} d \xi=o\left(\varepsilon^{2 \alpha-1}\right),
\end{aligned}
$$

where we have used Lebesgue's Dominated Convergence Theorem. We therefore have:

$$
E \approx \varepsilon^{\alpha-1}+\varepsilon^{\alpha-3}+\left(\lambda_{1}-\frac{4 \pi}{3} \lambda_{2}\right) \varepsilon^{2 \alpha-1}+o\left(\varepsilon^{2 \alpha-1}\right) .
$$

If we choose $\alpha<-2$, then the leading order term is the third one, and the lemma follows.

As a consequence we are now able to prove finite time blow-up for a certain class of initial data.

Theorem 5.2. Let $d=3$ and $\varphi \in \Sigma$. Denote $\underline{\omega}=\min \omega_{j}$ and assume that

$$
3 E \leqslant \underline{\omega}^{2}\|x \varphi\|_{L^{2}}^{2} .
$$

Then the solution $\psi$ blows up in finite time, i.e. $T_{*}<\infty$ in Corollary 4.1. More precisely, we can estimate $T_{*} \leqslant \pi /(2 \underline{\omega})$. 
From Lemma 5.1 we know that for $\lambda_{1}<\frac{4 \pi}{3} \lambda_{2}$ we can always choose initial data such that $E<0$ and thus enforce finite time blow-up. In other words, blow-up may occur even in situations where we have a defocusing (local) cubic nonlinearity $\left(\lambda_{1}>0\right)$ and a positive coupling constant $\lambda_{2}>0$ (the physical case), provided that $\lambda_{1}<\frac{4 \pi}{3} \lambda_{2}$ holds true (and the initial energy is sufficiently small). Recalling the well known fact that when $\lambda_{1}>0$ and $\lambda_{2}=0$, finite time blow-up cannot occur (i.e. $T_{*}=\infty$ ), this shows that the presence of the dipole-term may indeed cause collapse of the Bose-Einstein condensate.

Proof. The proof is based on the virial computation. Set

$$
y(t)=\int_{\mathbb{R}^{3}}|x|^{2}|\psi(t, x)|^{2} d x .
$$

Then, following [9] (with slightly different notations), we have, since $K$ is even:

$$
\begin{aligned}
\ddot{y}(t)= & 4 E+\lambda_{1}\|\psi(t)\|_{L^{4}}^{4}-8 \int_{\mathbb{R}^{3}} V(x)|\psi(t, x)|^{2} d x \\
& -2 \lambda_{2} \int_{\mathbb{R}^{3}}\left(\left(K+\frac{1}{2} x \cdot \nabla K\right) *|\psi|^{2}\right)(t, x)|\psi(t, x)|^{2} d x .
\end{aligned}
$$

We again use Plancherel's formula with $\rho=|\psi|^{2}$ to write

$$
\int\left(\left(K+\frac{1}{2} x \cdot \nabla K\right) * \rho\right)(t, x) \rho(t, x) d x=\frac{1}{(2 \pi)^{3}} \int\left(\widehat{K}+\frac{1}{2} \widehat{x \cdot \nabla K}\right)|\widehat{\rho}(\xi)|^{2} d \xi
$$

and compute, for $d=3$,

$$
\widehat{x \cdot \nabla K}(\xi)=-\operatorname{div}(\xi \widehat{K}(\xi))=-3 \widehat{K}(\xi)-\xi \cdot \nabla \widehat{K}(\xi) .
$$

From Lemma 2.3, we infer $\xi \cdot \nabla \widehat{K}(\xi)=0$ and thus

$$
\ddot{y}(t)=4 E+\frac{\lambda_{1}}{(2 \pi)^{3}}\|\widehat{\rho}(t)\|_{L^{2}}^{2}-8 \int_{\mathbb{R}^{3}} V(x)|\psi(t, x)|^{2} d x+\frac{\lambda_{2}}{(2 \pi)^{3}} \int_{\mathbb{R}^{3}} \widehat{K}(\xi)|\widehat{\rho}(\xi)|^{2} d \xi .
$$

This can be rewritten as

with $\underline{\omega}=\min \omega_{j}$ and

$$
\ddot{y}(t)+4 \underline{\omega}^{2} y(t)=f(t)
$$

$f(t)=4 E-4 \sum_{j=1}^{3}\left(\omega_{j}^{2}-\underline{\omega}^{2}\right) \int_{\mathbb{R}^{3}} x_{j}^{2}|\psi(t, x)|^{2} d x+\frac{1}{(2 \pi)^{3}} \int_{\mathbb{R}^{3}}\left(\lambda_{1}+\lambda_{2} \widehat{K}(\xi)\right)|\widehat{\rho}(\xi)|^{2} d \xi$.

Recalling the energy as written as in (5.1), we first note that the source term $f(t)$ can be estimated via

$$
\begin{aligned}
f(t) & \leqslant 4 E+\frac{1}{(2 \pi)^{3}} \int_{\mathbb{R}^{3}}\left(\lambda_{1}+\lambda_{2} \widehat{K}(\xi)\right)|\widehat{\rho}(\xi)|^{2} d \xi \\
& \leqslant 6 E-\|\nabla \psi(t)\|_{L^{2}}^{2}-2 \int_{\mathbb{R}^{3}} V(x)|\psi(t, x)|^{2} d x \leqslant 6 E .
\end{aligned}
$$

On the other hand we have

$$
y(t)=y(0) \cos (2 \underline{\omega} t)+\dot{y}(0) \frac{\sin (2 \underline{\omega} t)}{2 \underline{\omega}}+\int_{0}^{t} \frac{\sin (2 \underline{\omega}(t-s))}{2 \underline{\omega}} f(s) d s .
$$

Suppose now that $T_{*}>\pi /(2 \underline{\omega})$. Then we can consider $t=\pi /(2 \underline{\omega})$ in the above relation. This yields, since the sine function in the last integral remains non-negative on $[0, t]$ :

$$
y\left(\frac{\pi}{2 \underline{\omega}}\right) \leqslant-y(0)+6 E \int_{0}^{\pi /(2 \underline{\omega})} \frac{\sin (\pi-2 \underline{\omega} s)}{2 \underline{\omega}} d s=-y(0)+\frac{3 E}{\underline{\omega}^{2}} .
$$


We note that the left hand side must be positive, since $\psi(t, \cdot) \in \Sigma$. By assumption, the right hand side is non-positive. This yields a contradiction and hence the result.

Remark 5.3. To shed more light on the conditions for blow-up, consider the case of an isotropic trapping potential: $\omega_{j}=\omega$ for $1 \leqslant j \leqslant 3$. The condition of Theorem 5.2 then reads

$$
\frac{3}{2}\|\nabla \varphi\|_{L^{2}}^{2}+\frac{\omega^{2}}{2}\|x \varphi\|_{L^{2}}^{2}+\frac{3 \lambda_{1}}{2}\|\varphi\|_{L^{4}}^{4}+\frac{3 \lambda_{2}}{2} \int_{\mathbb{R}^{3}}\left(K_{d} *|\varphi|^{2}\right)(x)|\varphi(x)|^{2} d x \leqslant 0 .
$$

In view of Plancherel's formula, this is equivalent to

$$
3\|\nabla \varphi\|_{L^{2}}^{2}+\omega^{2}\|x \varphi\|_{L^{2}}^{2}+\frac{3}{(2 \pi)^{3}} \int_{\mathbb{R}^{3}}\left(\lambda_{1}+\lambda_{2} \widehat{K}(\xi)\right)|\widehat{\rho}(\xi)|^{2} d \xi \leqslant 0 .
$$

From Lemma 2.3, when $\lambda_{2}>0$ (the physical case), this is possible only if $\lambda_{1}<\frac{4 \pi}{3} \lambda_{2}$.

We already know that when $\lambda_{1} \geqslant \frac{4 \pi}{3} \lambda_{2}$, then finite time blow-up cannot occur. On the other hand we have just seen that blow-up occurs in the case $\lambda_{1}<\frac{4 \pi}{3} \lambda_{2}$, provided the initial energy is sufficiently small, say non-positive. What remains open therefore is the case of (large) positive initial energy in the unstable regime. Unfortunately we can only give a partial answer to that.

5.2. Global existence for $d=3$ (unstable regime). Here we shall show that global in time existence is possible in the case $\lambda_{1}<\frac{4 \pi}{3} \lambda_{2}$ under some additional assumptions.

Proposition 5.4. Under the same assumptions as in Proposition 3.4, $d=3$, suppose that in addition $\lambda_{1}<\frac{4 \pi}{3} \lambda_{2}$ and $\lambda_{2} \geqslant 0$. Then there exists a $C_{0}>0$, independent of $\lambda_{1}$ and $\lambda_{2}$, and a (positive) constant

$$
\widetilde{C}_{0}=\frac{C_{0}}{M\left(\frac{4 \pi}{3} \lambda_{2}-\lambda_{1}\right)^{2}},
$$

such that, if $0<E<\widetilde{C}_{0}$ and $\|\nabla \varphi\|_{L^{2}}^{2}<\widetilde{C}_{0}$, then $T_{*}=\infty$.

Of course, this result can only give an additional insight in situations where $\underline{\omega}^{2}\|x \varphi\|_{L^{2}}^{2}<3 \widetilde{C}_{0}$. The picture becomes a bit clearer, though, if one ignores the harmonic confinement for a moment, i.e. set $\omega_{j}=0$. Then we know that in the unstable regime $\lambda_{1}<\frac{4 \pi}{3} \lambda_{2}$ blow-up occurs as soon as the total initial energy is non-positive. On the other hand, if the total initial energy is positive but not too large, i.e. smaller than $\widetilde{C}_{0}$, then global in time existence still holds, provided the initial kinetic energy is also smaller than $\widetilde{C}_{0}$.

Proof. We resume the same approach as in the proof of Theorem 4.4, from which we now get

$\|\nabla \psi(t)\|_{L^{2}}^{2} \leqslant 2 E+\frac{1}{(2 \pi)^{3}} \int_{\mathbb{R}^{3}}\left(\frac{4 \pi}{3} \lambda_{2}-\lambda_{1}\right)|\widehat{\rho}(\xi)|^{2} d \xi=2 E+\left(\frac{4 \pi}{3} \lambda_{2}-\lambda_{1}\right)\|\psi(t)\|_{L^{4}}^{4}$.

The Gagliardo-Nirenberg inequality then yields

$$
\|\nabla \psi(t)\|_{L^{2}}^{2} \leqslant 2 E+C\left(\frac{4 \pi}{3} \lambda_{2}-\lambda_{1}\right)\|\psi(t)\|_{L^{2}}\|\nabla \psi(t)\|_{L^{2}}^{3} .
$$

On the other hand, the conservation of mass implies:

$$
\|\nabla \psi(t)\|_{L^{2}}^{2} \leqslant 2 E+C\left(\frac{4 \pi}{3} \lambda_{2}-\lambda_{1}\right) \sqrt{M}\|\nabla \psi(t)\|_{L^{2}}^{3} .
$$

With these two estimates in hand, the result follows from a bootstrap argument (see e.g. [2]): 
Lemma 5.5 (Bootstrap argument). Let $f=f(t)$ be a nonnegative continuous function on $[0, T]$ such that, for every $t \in[0, T]$,

$$
f(t) \leqslant \varepsilon_{1}+\varepsilon_{2} f(t)^{\theta}
$$

where $\varepsilon_{1}, \varepsilon_{2}>0$ and $\theta>1$ are constants such that

$$
\varepsilon_{1}<\left(1-\frac{1}{\theta}\right) \frac{1}{\left(\theta \varepsilon_{2}\right)^{1 /(\theta-1)}}, \quad f(0) \leqslant \frac{1}{\left(\theta \varepsilon_{2}\right)^{1 /(\theta-1)}} .
$$

Then, for every $t \in[0, T]$, we have

$$
f(t) \leqslant \frac{\theta}{\theta-1} \varepsilon_{1} .
$$

We apply the lemma with $f(t)=\|\nabla \psi(t)\|_{L^{2}}^{2}, \varepsilon_{1}=2 E, \varepsilon_{2}=C\left(\frac{4 \pi}{3} \lambda_{2}-\lambda_{1}\right) \sqrt{M}$ and $\theta=3 / 2$. Note that we therefore have to assume that the energy is positive. Otherwise, (5.2) yields no information any way, and Theorem 5.2 shows that finite time blow-up occurs.

\section{DiMENSION REDUCTION}

We shall mainly follow the ideas of [5] where such an analysis has been rigorously performed for $\lambda_{2}=0$ (no dipole effects) in the case of modulated ground state intial data. We also remark that the case of general initial data has been treated in the remarkable paper [4]. In our case, due to the presence of the non-isotropic dipolekernel, we can distinguish two main cases: The reduction from $d=3$ to an effective one-dimensional model in the dipole direction $n=(0,0,1)$, and the reduction to an effective two-dimensional model perpendicular to $n$. We shall sketch the adaptation of the approach in [5] to the present context. It is very likely that the analysis of [4] can be adapted to the dipole case, but we shall not pursue this question as it is beyond the scope of our work.

6.1. Formal derivation of the one-dimensional model. Let us start with the first problem of deriving an effective one-dimensional model in the dipole direction. To this end we write the general model (3.1) in the form

$$
i \partial_{t} \psi+\frac{1}{2} \Delta \psi=\frac{1}{\varepsilon^{4}}\left(\omega_{1}^{2} \frac{x_{1}^{2}}{2}+\omega_{2}^{2} \frac{x_{2}^{2}}{2}\right) \psi+\omega_{3}^{2} \frac{x_{3}^{2}}{2} \psi+\lambda_{1}|\psi|^{2} \psi+\lambda_{2}\left(K *|\psi|^{2}\right) \psi
$$

The parameter $\varepsilon$ is positive and small, its smallness modeling a strong confinement in the first two directions. Introduce a change of variables via

$$
\psi\left(t, x_{1}, x_{2}, x_{3}\right)=\psi^{\varepsilon}\left(t, \frac{x_{1}}{\varepsilon}, \frac{x_{2}}{\varepsilon}, x_{3}\right) .
$$

Then equation (6.1) is equivalent to:

$$
i \partial_{t} \psi^{\varepsilon}+\frac{1}{2} \frac{\partial^{2}}{\partial x_{3}^{2}} \psi^{\varepsilon}=\frac{1}{\varepsilon^{2}} H_{2} \psi^{\varepsilon}+\omega_{3}^{2} \frac{x_{3}^{2}}{2} \psi^{\varepsilon}+\lambda_{1}\left|\psi^{\varepsilon}\right|^{2} \psi^{\varepsilon}+\lambda_{2} \mathcal{K}^{\varepsilon} \psi^{\varepsilon},
$$

where $\mathrm{H}_{2}$ is the two-dimensional harmonic oscillator

$$
H_{2}=-\frac{1}{2}\left(\frac{\partial^{2}}{\partial x_{1}^{2}}+\frac{\partial^{2}}{\partial x_{2}^{2}}\right)+\omega_{1}^{2} \frac{x_{1}^{2}}{2}+\omega_{2}^{2} \frac{x_{2}^{2}}{2},
$$

and $\mathcal{K}^{\varepsilon}$ is the convolution operator

$$
\mathcal{K}^{\varepsilon}(t, x)=\int_{\mathbb{R}^{3}} \frac{\left(\varepsilon x_{1}-y_{1}\right)^{2}+\left(\varepsilon x_{2}-y_{2}\right)^{2}-2\left(x_{3}-y_{3}\right)^{2}}{\left(\left(\varepsilon x_{1}-y_{1}\right)^{2}+\left(\varepsilon x_{2}-y_{2}\right)^{2}+\left(x_{3}-y_{3}\right)^{2}\right)^{5 / 2}}\left|\psi^{\varepsilon}(t, y)\right|^{2} d y .
$$


For $\ell \in \mathbb{N}$, we shall denote by $\chi_{\ell}\left(x_{1}, x_{2}\right)$ the (normalized) eigenfunction of $H_{2}$ corresponding to the eigenvalue $\mu_{\ell} \in \mathbb{R}_{+}$. In particular the ground state $\chi_{0}$ corresponds to $\mu_{0}=\frac{1}{2}\left(\omega_{1}+\omega_{2}\right)$ and is explicitly given by

$$
\chi_{0}\left(x_{1}, x_{2}\right)=\frac{\sqrt{\omega_{1} \omega_{2}}}{\pi} e^{-\left(\omega_{1} x_{1}^{2}+\omega_{2} x_{2}^{2}\right) / 2} .
$$

As in [5], we consequently seek a solution to (6.2) in the form

$$
\psi^{\varepsilon}\left(t, x_{1}, x_{2}, x_{3}\right)=e^{-i \mu_{0} t / \varepsilon^{2}} \chi_{0}\left(x_{1}, x_{2}\right) u\left(t, x_{3}\right) .
$$

This type of ansatz obviously requires well-prepared initial data (i.e. concentrated on the ground state of $H_{2}$ ). A formal multiple scales expansion for $\psi^{\varepsilon}$ as $\varepsilon \rightarrow 0$ then yields the consistency relation

$$
\left(H_{2}-\mu_{0}\right) \psi^{\varepsilon}=0
$$

plus an evolution equation for the modulation $u(t, x)$, given by

$$
i \partial_{t} u+\frac{1}{2} \frac{\partial^{2}}{\partial x_{3}^{2}} u=\omega_{3}^{2} \frac{x_{3}^{2}}{2} u+\kappa_{1}|u|^{2} u+\lambda_{2}\left(K_{1} *|u|^{2}\right) u,
$$

Here we denote by

$$
\kappa_{1}=\lambda_{1} \int_{\mathbb{R}^{2}} \chi_{0}^{4}\left(x_{1}, x_{2}\right) d x_{1} d x_{2},
$$

the effective coupling constant for the cubic nonlinearity and by

$$
K_{1}\left(x_{3}\right)=\int_{\mathbb{R}^{2}} \frac{x_{1}^{2}+x_{2}^{2}-2 x_{3}^{2}}{\left(x_{1}^{2}+x_{2}^{2}+x_{3}^{2}\right)^{5 / 2}} \chi_{0}\left(x_{1}, x_{2}\right)^{2} d x_{1} d x_{2} .
$$

the corresponding effective one-dimensional dipole kernel. To apply the existence analysis presented in $\S 4$, it is important to check if the operator $\mathcal{K}_{1}: u \mapsto K_{1} * u$ is bounded on $L^{2}(\mathbb{R})$. To do so we shall show that the one-dimensional Fourier transform of $K_{1}$ is in $L^{\infty}(\mathbb{R})$. Recall

$$
K\left(x_{1}, x_{2}, x_{3}\right)=\frac{x_{1}^{2}+x_{2}^{2}-2 x_{3}^{2}}{\left(x_{1}^{2}+x_{2}^{2}+x_{3}^{2}\right)^{5 / 2}} .
$$

Then, denoting by $\mathcal{F}_{2}$ the partial Fourier transform with respect to the variables $x_{1}$ and $x_{2}$ only, we obtain from Plancherel's formula on $\mathbb{R}^{2}$, that

$$
\begin{aligned}
\widehat{K}_{1}\left(\xi_{3}\right) & =\int_{\mathbb{R}} e^{-i x_{3} \xi_{3}} K_{1}\left(x_{3}\right) d x_{3} \\
& =\int_{\mathbb{R}^{3}} e^{-i x_{3} \xi_{3}} K\left(x_{1}, x_{2}, x_{3}\right) \overline{\chi_{0}\left(x_{1}, x_{2}\right)^{2}} d x_{1} d x_{2} d x_{3} \\
& =\frac{1}{(2 \pi)^{2}} \int_{\mathbb{R}^{3}} e^{-i x_{3} \xi_{3}} \mathcal{F}_{2} K\left(\xi_{1}, \xi_{2}, x_{3}\right) \overline{\mathcal{F}_{2} \chi_{0}^{2}}\left(\xi_{1}, \xi_{2}\right) d \xi_{1} d \xi_{2} d x_{3} \\
& =\frac{1}{(2 \pi)^{2}} \int_{\mathbb{R}^{2}} \overline{\mathcal{F}_{2} \chi_{0}^{2}}\left(\xi_{1}, \xi_{2}\right) d \xi_{1} d \xi_{2} \int_{\mathbb{R}} e^{-i x_{3} \xi_{3}} \mathcal{F}_{2} K\left(\xi_{1}, \xi_{2}, x_{3}\right) d x_{3} \\
& =\frac{1}{(2 \pi)^{2}} \int_{\mathbb{R}^{3}} \overline{\mathcal{F}_{2} \chi_{0}^{2}}\left(\xi_{1}, \xi_{2}\right) \widehat{K}\left(\xi_{1}, \xi_{2}, \xi_{3}\right) d \xi_{1} d \xi_{2} d \xi_{3},
\end{aligned}
$$

where $\widehat{K}$ denotes the three-dimensional Fourier transform of the kernel $K$. Since $\chi_{0}$ is a Schwartz function, the Fourier transform of $\chi_{0}^{2}$ is a Schwartz function, thereby in $L^{1}(\mathbb{R})$. Consequently, the boundedness of $\widehat{K}$ on $\mathbb{R}^{3}$ (which stems from Lemma 2.1, or more explicitly from Lemma 2.3 ) implies the boundedness of $\widehat{K}_{1}$ on $\mathbb{R}$.

The assumptions of Proposition 3.4 are thus satisfied. From Corollary 4.2, we know that the effective one-dimensional model (6.4) has a unique, global solution in $\Sigma$. Moreover, the same holds true if $\Sigma$ is replaced by $L^{2}(\mathbb{R})$, as proved in Theorem 4.3. 
6.2. Formal derivation of the two-dimensional model. We proceed as before. Starting from

$$
i \partial_{t} \psi+\frac{1}{2} \Delta \psi=\left(\omega_{1}^{2} \frac{x_{1}^{2}}{2}+\omega_{2}^{2} \frac{x_{2}^{2}}{2}\right) \psi+\frac{\omega_{3}^{2}}{\varepsilon^{4}} \frac{x_{3}^{2}}{2} \psi+\lambda_{1}|\psi|^{2} \psi+\lambda_{2}\left(K *|\psi|^{2}\right) \psi,
$$

we introduce a new change of variables by

$$
\psi\left(t, x_{1}, x_{2}, x_{3}\right)=\widetilde{\psi}^{\varepsilon}\left(t, x_{1}, x_{2}, \frac{x_{3}}{\varepsilon}\right) .
$$

Equation (6.6) then becomes

$i \partial_{t} \widetilde{\psi}^{\varepsilon}+\frac{1}{2}\left(\frac{\partial^{2}}{\partial x_{1}^{2}}+\frac{\partial^{2}}{\partial x_{2}^{2}}\right) \widetilde{\psi}^{\varepsilon}=\frac{1}{\varepsilon^{2}} H_{1} \widetilde{\psi}^{\varepsilon}+\frac{1}{2}\left(\omega_{1}^{2} x_{1}^{2}+\omega_{2}^{2} x_{2}^{2}\right) \widetilde{\psi}^{\varepsilon}+\lambda_{1}\left|\widetilde{\psi}^{\varepsilon}\right|^{2} \widetilde{\psi}^{\varepsilon}+\lambda_{2} \widetilde{\mathcal{K}}^{\varepsilon} \widetilde{\psi}^{\varepsilon}$,

where $H_{1}$ now denotes the one-dimensional harmonic oscillator, acting in the dipole direction

$$
H_{1}=-\frac{\partial^{2}}{\partial x_{3}^{2}}+\omega_{3}^{2} \frac{x_{3}^{2}}{2} .
$$

Denoting the corresponding ground state by $\tilde{\chi}_{0}\left(x_{3}\right)$ and proceeding analogously as before we arrive, instead of (6.2), at the following effective equation for the modulation $\widetilde{u}\left(t, x_{1}, x_{2}\right)$ :

$$
i \partial_{t} \widetilde{u}+\frac{1}{2}\left(\frac{\partial^{2}}{\partial x_{1}^{2}}+\frac{\partial^{2}}{\partial x_{2}^{2}}\right) \widetilde{u}=\frac{1}{2}\left(\omega_{1}^{2} x_{1}^{2}+\omega_{2}^{2} x_{2}^{2}\right) \widetilde{u}+\widetilde{\kappa}_{1}|\widetilde{u}|^{2} \widetilde{u}+\lambda_{2} K_{2} \widetilde{u},
$$

where now

$$
\widetilde{\kappa}_{1}=\lambda_{1} \int_{\mathbb{R}} \widetilde{\chi}_{0}^{4}\left(x_{3}\right) d x_{3}
$$

is the new coupling constant and

$$
K_{2}\left(x_{1}, x_{2}\right)=\int_{\mathbb{R}} \frac{x_{1}^{2}+x_{2}^{2}-2 x_{3}^{2}}{\left(x_{1}^{2}+x_{2}^{2}+x_{3}^{2}\right)^{5 / 2}} \chi_{0}\left(x_{3}\right)^{2} d x_{3} .
$$

is the effective two-dimensional dipole kernel. By the same computation as above, we see that $\mathcal{F}_{2} K_{2} \in L^{\infty}\left(\mathbb{R}^{2}\right)$, and thus the assumptions of Proposition 3.4 are satisfied to guarantee a well-posed initial value problem in $\Sigma$, at least locally in time.

6.3. A rigorous result. For completeness we shall finally state a rigorous mathematical result and sketch the corresponding proof which follows the lines of [5]. We only consider the case $\lambda_{1} \geqslant \frac{4 \pi}{3} \lambda_{2} \geqslant 0$ (the stable regime), which allows for a somewhat shorter argument and again refer to [5, 4] for more general statements. Moreover, for notational convenience, we shall state the result only for the case of (6.4) (one-dimensional model in dipole direction), but exactly the same approach can be followed for justifying (6.7). We consider initial data for $\psi^{\varepsilon}$ which do not depend on $\varepsilon$, and refer to $[5,4]$ for a discussion on this assumption.

Theorem 6.1. Let $d=3, V_{d}$ be quadratic and $\lambda_{1} \geqslant \frac{4 \pi}{3} \lambda_{2} \geqslant 0$. Denote by $\psi^{\varepsilon}(t) \in \Sigma$ and $u(t) \in \Sigma$ the unique solutions of (6.2) and (6.4), respectively, with

$$
\left.\psi^{\varepsilon}\right|_{t=0}=\chi_{0}\left(x_{1}, x_{2}\right) u_{0}\left(x_{3}\right),\left.\quad u\right|_{t=0}=u_{0}\left(x_{3}\right), \quad u_{0} \in \Sigma .
$$

Then, for any $T<\infty$, there exists $C_{T}>0$ such that

$$
\sup _{t \in[0, T]}\left\|\psi^{\varepsilon}(t)-e^{-i \mu_{0} t / \varepsilon^{2}} u(t) \chi_{0}\right\|_{L^{2}\left(\mathbb{R}^{3}\right)} \leqslant C_{T} \varepsilon \text {. }
$$


Proof (Sketch). The energy associated to (6.2) can be written as

$$
\begin{aligned}
E= & \left\langle\psi^{\varepsilon}(t), H_{1} \psi^{\varepsilon}(t)\right\rangle+\frac{1}{\varepsilon^{2}}\left\langle\psi^{\varepsilon}(t), H_{2} \psi^{\varepsilon}(t)\right\rangle \\
& +\frac{1}{2(2 \pi)^{3}} \int_{\mathbb{R}^{3}}\left(\lambda_{1}+\frac{4 \pi}{3} \lambda_{2}\left(3 \frac{\xi_{3}^{2}}{|\xi|^{2}}-1\right)\right)|\widehat{\rho}(\xi)|^{2} d \xi
\end{aligned}
$$

where $\langle\cdot, \cdot\rangle$ denotes the scalar product in $L^{2}\left(\mathbb{R}^{3}\right)$, and $H_{1}, H_{2}$ denote the onedimensional and two-dimensional harmonic oscillator operator, respectively. In the following we denote by $\Pi_{\ell}$ the orthogonal projector onto the eigenspace corresponding to $\chi_{\ell}\left(x_{3}\right)$ and define

$$
\psi_{\ell}^{\varepsilon}\left(t, x_{3}\right)=e^{i \mu_{0} t / \varepsilon^{2}} \int_{\mathbb{R}^{2}} \psi^{\varepsilon}\left(t, x_{1}, x_{2}, x_{3}\right) \chi_{\ell}\left(x_{1}, x_{2}\right) d x_{1} d x_{2} .
$$

Recalling the ansatz (6.3) for $\psi^{\varepsilon}(t)$ and using the fact that the eigenfunctions $\left\{\chi_{\ell}\right\}_{\ell \in \mathbb{N}}$ form an orthonormal basis of $L^{2}\left(\mathbb{R}^{2}\right)$ we note that $\chi_{0 \mid t=0}^{\varepsilon}=u_{\mid t=0}$, since $\left\|\chi_{0}\right\|_{L^{2}}=1$. Thus, we can rewrite

$$
\left\langle\psi^{\varepsilon}(t), H_{2} \psi^{\varepsilon}(t)\right\rangle=\sum_{\ell=1}^{\infty}\left(\mu_{\ell}-\mu_{0}\right)\left\|\psi_{\ell}^{\varepsilon}(t)\right\|_{L^{2}}^{2}+\mu_{0}\|u(0)\|_{L^{2}}^{2} .
$$

Keeping in mind that, by assumption $\left\langle\psi^{\varepsilon}(0), H_{2} \psi^{\varepsilon}(0)\right\rangle=\mu_{0}\|u(0)\|_{L^{2}(\mathbb{R})}^{2}$, the conservation of energy implies

$$
\begin{aligned}
E_{\text {red }}= & \left\langle\psi^{\varepsilon}(t), H_{1} \psi^{\varepsilon}(t)\right\rangle+\frac{1}{\varepsilon^{2}} \sum_{\ell=1}^{\infty}\left(\mu_{\ell}-\mu_{0}\right)\left\|\psi_{\ell}^{\varepsilon}(t)\right\|_{L^{2}}^{2} \\
& +\frac{1}{2(2 \pi)^{3}} \int_{\mathbb{R}^{3}}\left(\lambda_{1}+\frac{4 \pi}{3} \lambda_{2}\left(3 \frac{\xi_{3}^{2}}{|\xi|^{2}}-1\right)\right)|\widehat{\rho}(\xi)|^{2} d \xi,
\end{aligned}
$$

where the reduced initial energy is

$$
\begin{aligned}
E_{\mathrm{red}} & =\left\langle\psi^{\varepsilon}(0), H_{1} \psi^{\varepsilon}(0)\right\rangle+\frac{1}{2(2 \pi)^{3}} \int_{\mathbb{R}^{3}}\left(\lambda_{1}+\frac{4 \pi}{3} \lambda_{2}\left(3 \frac{\xi_{3}^{2}}{|\xi|^{2}}-1\right)\right)|\widehat{\rho}(\xi)|^{2} d \xi \\
& \geqslant\left\langle\psi^{\varepsilon}(0), H_{1} \psi^{\varepsilon}(0)\right\rangle+\frac{1}{2(2 \pi)^{3}} \int_{\mathbb{R}^{3}}\left(\lambda_{1}-\frac{4 \pi}{3} \lambda_{2}\right)|\widehat{\rho}(\xi)|^{2} d \xi \geqslant 0,
\end{aligned}
$$

by assumption on the parameters $\lambda_{1}, \lambda_{2}$. On the other hand, by exactly the same argument we infer from the energy conservation above that

$$
\begin{aligned}
E_{\text {red }} \geqslant & \left\langle\psi^{\varepsilon}(t), H_{1} \psi^{\varepsilon}(t)\right\rangle+\frac{1}{\varepsilon^{2}} \sum_{\ell=1}^{\infty}\left(\mu_{\ell}-\mu_{0}\right)\left\|\psi_{\ell}^{\varepsilon}(t)\right\|_{L^{2}}^{2} \\
& +\frac{1}{2(2 \pi)^{3}} \int_{\mathbb{R}^{3}}\left(\lambda_{1}-\frac{4 \pi}{3} \lambda_{2}\right)|\widehat{\rho}(\xi)|^{2} d \xi .
\end{aligned}
$$

All the terms in this equation are non-negative and thus, we immediately obtain

$$
\sum_{\ell=1}^{\infty}\left(\mu_{\ell}-\mu_{0}\right)\left\|\psi_{\ell}^{\varepsilon}(t)\right\|_{L^{2}}^{2} \leqslant C \varepsilon^{2} .
$$

Denoting by $\Pi_{0}$ the projection onto the eigenspace generated by the ground state $\chi_{0}$, we can thus estimate, since $\psi_{0}^{\varepsilon} \chi_{0}=e^{i \mu_{0} t / \varepsilon^{2}} \Pi_{0} \psi^{\varepsilon}$,

$$
\begin{aligned}
& \left\|\psi^{\varepsilon}(t)-e^{-i \mu_{0} t / \varepsilon^{2}} \chi_{0} u(t)\right\|_{L^{2}\left(\mathbb{R}^{3}\right)} \\
& \leqslant\left\|\left(1-\Pi_{0}\right) \psi^{\varepsilon}(t)\right\|_{L^{2}\left(\mathbb{R}^{3}\right)}+\left\|e^{-i \mu_{0} t / \varepsilon^{2}} \chi_{0}\left(\psi_{0}^{\varepsilon}(t)-u(t)\right)\right\|_{L^{2}\left(\mathbb{R}^{3}\right)} \\
& =\left\|\left(1-\Pi_{0}\right) \psi^{\varepsilon}(t)\right\|_{L^{2}\left(\mathbb{R}^{3}\right)}+\left\|\psi_{0}^{\varepsilon}(t)-u(t)\right\|_{L^{2}(\mathbb{R})} .
\end{aligned}
$$


For the first term on the right hand side, we have

$$
\left\|\left(1-\Pi_{0}\right) \psi^{\varepsilon}(t)\right\|_{L^{2}\left(\mathbb{R}^{3}\right)}^{2}=\sum_{\ell=1}^{\infty}\left\|\psi_{\ell}^{\varepsilon}(t)\right\|_{L^{2}}^{2} \leqslant \frac{1}{\mu_{1}-\mu_{0}} \sum_{\ell=1}^{\infty}\left(\mu_{\ell}-\mu_{0}\right)\left\|\psi_{\ell}^{\varepsilon}(t)\right\|_{L^{2}}^{2} \leqslant C \varepsilon^{2} .
$$

Applying one derivative with respect to $x_{1}$ or $x_{2}$, Sobolev embedding shows that the above estimate remains valid if the $L^{2}\left(\mathbb{R}^{3}\right)$-norm is replaced by $L_{x_{3}}^{2}\left(L_{x_{1}, x_{2}}^{p}\right)$, for $2 \leqslant p<\infty$.

For the term $\psi_{0}^{\varepsilon}-u$, more computations are necessary, and we shall emphasize the main arguments only. First, as in [5], we check that $\psi_{0}^{\varepsilon}$ solves

$$
i \partial_{t} \psi_{0}^{\varepsilon}=H_{1} \psi_{0}^{\varepsilon}+F_{1}^{\varepsilon},
$$

where

$$
F_{1}^{\varepsilon}\left(t, x_{3}\right)=e^{i \mu_{0} t / \varepsilon^{2}} \int_{\mathbb{R}^{2}}\left(\lambda_{1}\left|\psi^{\varepsilon}\right|^{2} \psi^{\varepsilon}+\lambda_{2} \mathcal{K}^{\varepsilon} \psi^{\varepsilon}\right) \chi_{0}\left(x_{1}, x_{2}\right) d x_{1} d x_{2} .
$$

Recall that $u$ solves

$$
i \partial_{t} u=H_{1} u+\lambda_{1}|u|^{2} u \int_{\mathbb{R}^{2}} \chi_{0}^{4}\left(x_{1}, x_{2}\right) d x_{1} d x_{2}+\lambda_{2}\left(K_{1} *|u|^{2}\right) u,
$$

where $K_{1}$ is given by (6.5). Introducing the error $w^{\varepsilon}=\psi_{0}^{\varepsilon}-u$, we can write

$$
i \partial_{t} w^{\varepsilon}=H_{1} w^{\varepsilon}+L^{\varepsilon}+\lambda_{1} S_{1}^{\varepsilon}+\lambda_{2} S_{2}^{\varepsilon},
$$

where

$$
\begin{aligned}
L^{\varepsilon} & =\lambda_{1}\left(\left|\psi_{0}^{\varepsilon}\right|^{2} \psi_{0}^{\varepsilon}-|u|^{2} u\right) \int_{\mathbb{R}^{2}} \chi_{0}^{4}+\lambda_{2}\left(\left(K_{1} *\left|\psi_{0}^{\varepsilon}\right|^{2}\right) \chi_{0}^{\varepsilon}-\left(K_{1} *|u|^{2}\right) u\right), \\
S_{1}^{\varepsilon} & =e^{i \mu_{0} t / \varepsilon^{2}}\left(\Pi_{0}\left(\left|\psi^{\varepsilon}\right|^{2} \psi^{\varepsilon}\right)-\left|\Pi_{0} \psi^{\varepsilon}\right|^{2} \Pi_{0} \psi^{\varepsilon}\right), \\
S_{2}^{\varepsilon} & =e^{i \mu_{0} t / \varepsilon^{2}} \Pi_{0}\left(\mathcal{K}^{\varepsilon} \psi^{\varepsilon}\right)-\left(K_{1} *\left|\psi_{0}^{\varepsilon}\right|^{2}\right) \psi_{0}^{\varepsilon} .
\end{aligned}
$$

The end of the proof relies essentially on the same estimates as in the contraction part of the proof of Proposition 3.4 in the case $d=1$, based on Strichartz estimates; we refer to [5] for adaptations due to the fact that the variables $x_{1}$ and $x_{2}$ appear as parameters here. Roughly speaking, the term $L^{\varepsilon}$ is treated like a linear term in a Gronwall lemma, even though this not so clear unless one goes through the details of computation (which we shall not do): for tiny time intervals, the term corresponding to $L^{\varepsilon}$ is "absorbed by the left hand side". Since we consider only finite time intervals, we proceed this way a finite number of times, so the size of $w^{\varepsilon}$ is dictated by the size of the source terms $S_{1}^{\varepsilon}$ and $S_{2}^{\varepsilon}$ in suitable mixed time-space norms.

In view of the above remark on the estimate for $\left(1-\Pi_{0}\right) \psi^{\varepsilon}, S_{1}^{\varepsilon}$ is $\mathcal{O}(\varepsilon)$ in the spaces we need; this point is exactly the same as in [5], so we leave it out. To conclude, we simply consider the case of $S_{2}^{\varepsilon}$. Recall

$$
\mathcal{K}^{\varepsilon}=\int_{\mathbb{R}^{3}} \frac{\left(\varepsilon x_{1}-y_{1}\right)^{2}+\left(\varepsilon x_{2}-y_{2}\right)^{2}-2\left(x_{3}-y_{3}\right)^{2}}{\left(\left(\varepsilon x_{1}-y_{1}\right)^{2}+\left(\varepsilon x_{2}-y_{2}\right)^{2}+\left(x_{3}-y_{3}\right)^{2}\right)^{5 / 2}}\left|\psi^{\varepsilon}(t, y)\right|^{2} d y .
$$

Write

$$
\left|\psi^{\varepsilon}(t, y)\right|^{2}=\left|\psi^{\varepsilon}(t, y)\right|^{2}-\left|\Pi_{0} \psi^{\varepsilon}(t, y)\right|^{2}+\left|\Pi_{0} \psi^{\varepsilon}(t, y)\right|^{2} .
$$

Up to an error of order $\mathcal{O}(\varepsilon)$, we can replace $\mathcal{K}^{\varepsilon}$ by

$$
\mathcal{K}_{1}^{\varepsilon}=\int_{\mathbb{R}^{3}} \frac{\left(\varepsilon x_{1}-y_{1}\right)^{2}+\left(\varepsilon x_{2}-y_{2}\right)^{2}-2\left(x_{3}-y_{3}\right)^{2}}{\left(\left(\varepsilon x_{1}-y_{1}\right)^{2}+\left(\varepsilon x_{2}-y_{2}\right)^{2}+\left(x_{3}-y_{3}\right)^{2}\right)^{5 / 2}}\left|\psi_{0}^{\varepsilon}\left(t, y_{3}\right) \chi_{0}\left(y_{1}, y_{2}\right)\right|^{2} d y
$$

Essentially, the only new term to estimate is

$$
\Pi_{0} \mathcal{K}_{1}^{\varepsilon}-K_{1} *\left|\Pi_{0} \psi^{\varepsilon}\right|^{2}=\Pi_{0} \mathcal{K}_{1}^{\varepsilon}-K_{1} *\left|\psi_{0}^{\varepsilon} \chi_{0}\right|^{2} .
$$


Up to an extra "Gronwall term", we can replace this by $\Pi_{0} \widetilde{\mathcal{K}}_{1}^{\varepsilon}-K_{1} *\left|u \chi_{0}\right|^{2}$, where

$$
\widetilde{\mathcal{K}}_{1}^{\varepsilon}=\int_{\mathbb{R}^{3}} \frac{\left(\varepsilon x_{1}-y_{1}\right)^{2}+\left(\varepsilon x_{2}-y_{2}\right)^{2}-2\left(x_{3}-y_{3}\right)^{2}}{\left(\left(\varepsilon x_{1}-y_{1}\right)^{2}+\left(\varepsilon x_{2}-y_{2}\right)^{2}+\left(x_{3}-y_{3}\right)^{2}\right)^{5 / 2}}\left|u\left(t, y_{3}\right) \chi_{0}\left(y_{1}, y_{2}\right)\right|^{2} d y
$$

The difference $\Pi_{0} \widetilde{\mathcal{K}}_{1}^{\varepsilon}-K_{1} *|u \varphi|^{2}=: I^{\varepsilon}\left(t, x_{3}\right)$ reads

$$
I^{\varepsilon}=\int_{\mathbb{R}^{5}} \delta^{\varepsilon}\left(x_{1}, x_{2}, x_{3}, y_{1}, y_{2}, y_{3}\right)\left|u\left(t, y_{3}\right)\right|^{2} \chi_{0}\left(y_{1}, y_{2}\right)^{2} \chi_{0}\left(x_{1}, x_{2}\right)^{2} d y_{1} d y_{2} d y_{3} d x_{1} d x_{2}
$$

where

$$
\begin{aligned}
\delta^{\varepsilon} & =K\left(y_{1}-\varepsilon x_{1}, y_{2}-\varepsilon x_{2}, y_{3}-x_{3}\right)-K\left(y_{1}, y_{2}, y_{3}-x_{3}\right) \\
& =\frac{\left(\varepsilon x_{1}-y_{1}\right)^{2}+\left(\varepsilon x_{2}-y_{2}\right)^{2}-2\left(x_{3}-y_{3}\right)^{2}}{\left(\left(\varepsilon x_{1}-y_{1}\right)^{2}+\left(\varepsilon x_{2}-y_{2}\right)^{2}+\left(x_{3}-y_{3}\right)^{2}\right)^{5 / 2}}-\frac{y_{1}^{2}+y_{2}^{2}-2\left(x_{3}-y_{3}\right)^{2}}{\left(y_{1}^{2}+y_{2}^{2}+\left(x_{3}-y_{3}\right)^{2}\right)^{5 / 2}} .
\end{aligned}
$$

Plancherel's formula with respect to the variables $y_{1}$ and $y_{2}$ yields:

$$
\begin{aligned}
I^{\varepsilon}\left(t, x_{3}\right)=\frac{1}{(2 \pi)^{2}} \int_{\mathbb{R}^{5}} & \left(e^{-i \varepsilon\left(x_{1} \eta_{1}+x_{2} \eta_{2}\right)}-1\right) \mathcal{F}_{2} K\left(\eta_{1}, \eta_{2}, y_{3}-x_{3}\right) \overline{\mathcal{F}_{2}\left(\chi_{0}^{2}\right)}\left(\eta_{1}, \eta_{2}\right) \\
& \times\left|u\left(t, y_{3}\right)\right|^{2} \chi_{0}\left(x_{1}, x_{2}\right)^{2} d \eta_{1} d \eta_{2} d y_{3} d x_{1} d x_{2} .
\end{aligned}
$$

Now computing the $L^{2}$ norm of $I^{\varepsilon}$ (as a function of $x_{3}$ ), the boundedness of the three-dimensional Fourier transform of $K$ and the boundedness of $u$ in $L^{2}(\mathbb{R})$ yield $\left\|I^{\varepsilon}(t)\right\|_{L^{2}}^{2} \leqslant C \int_{\mathbb{R}^{4}}\left|e^{-i \varepsilon\left(x_{1} \eta_{1}+x_{2} \eta_{2}\right)}-1\right|^{2}\left|\mathcal{F}_{2}\left(\chi_{0}^{2}\right)\left(\eta_{1}, \eta_{2}\right) \chi_{0}\left(x_{1}, x_{2}\right)^{2}\right|^{2} d \eta_{1} d \eta_{2} d x_{1} d x_{2}$.

Recalling that $\chi_{0} \in \mathcal{S}\left(\mathbb{R}^{2}\right)$, the standard estimate $\left|e^{i \theta}-1\right| \leqslant|\theta|$ for $\theta \in \mathbb{R}$ shows that the $L^{2}$ norm of $I^{\varepsilon}$ is $\mathcal{O}(\varepsilon)$, uniformly for $t \geqslant 0$. Therefore, its $L^{1}\left([0, T] ; L^{2}\right)$ norm (the norm that appears in energy estimates, which are a particular case of Strichartz estimates) is of order $\mathcal{O}(\varepsilon T)$, and the proposition follows.

\section{REFERENCES}

1. M. Abramowitz and I. A. Stegun, Handbook of mathematical functions with formulas, graphs, and mathematical tables, National Bureau of Standards Applied Mathematics Series, vol. 55, For sale by the Superintendent of Documents, U.S. Government Printing Office, Washington, D.C., 1964.

2. H. Bahouri and P. Gérard, High frequency approximation of solutions to critical nonlinear wave equations, Amer. J. Math. 121 (1999), no. 1, 131-175.

3. W. Bao, D. Jaksch, and P. A. Markowich, Numerical solution of the Gross-Pitaevskii equation for Bose-Einstein condensation, J. Comput. Phys. 187 (2003), no. 1, 318-342.

4. N. Ben Abdallah, F. Castella, and F. Méhats, Time averaging for the strongly confined nonlinear Schrödinger equation, using almost periodicity, J. Differential Equations (2008), to appear.

5. N. Ben Abdallah, F. Méhats, C. Schmeiser, and R. M. Weishäupl, The nonlinear Schrödinger equation with a strongly anisotropic harmonic potential, SIAM J. Math. Anal. 37 (2005), no. 1, 189-199.

6. R. Carles, Linear vs. nonlinear effects for nonlinear Schrödinger equations with potential, Commun. Contemp. Math. 7 (2005), no. 4, 483-508.

7. - On instability for the cubic nonlinear Schrödinger equation, C. R. Math. Acad. Sci. Paris 344 (2007), no. 8, 483-486.

8. - On the Cauchy problem in Sobolev spaces for nonlinear Schrödinger equations with potential, Portugal. Math. (N. S.) 65 (2008), no. 2, 191-209.

9. T. Cazenave, Semilinear Schrödinger equations, Courant Lecture Notes in Mathematics, vol. 10, New York University Courant Institute of Mathematical Sciences, New York, 2003.

10. R. P. Feynman and A. R. Hibbs, Quantum mechanics and path integrals (International Series in Pure and Applied Physics), Maidenhead, Berksh.: McGraw-Hill Publishing Company, Ltd., 365 p., 1965.

11. J. Ginibre and G. Velo, Scattering theory in the energy space for a class of nonlinear Schrödinger equations, J. Math. Pures Appl. (9) 64 (1985), no. 4, 363-401. 
12. R. T. Glassey, On the blowing up of solutions to the Cauchy problem for nonlinear Schrödinger equations, J. Math. Phys. 18 (1977), 1794-1797.

13. K. Góral and L. Santos, Ground state and elementary excitations of single and binary boseeinstein condensates of trapped dipolar gases, Phys. Rev. A 66 (2002), no. 2, 023613.

14. T. Kato, On nonlinear Schrödinger equations, Ann. IHP (Phys. Théor.) 46 (1987), no. 1, 113-129.

15. M. Keel and T. Tao, Endpoint Strichartz estimates, Amer. J. Math. 120 (1998), no. 5, 955980.

16. S. Ronen, D. C. E. Bortolotti, D. Blume, and J. L. Bohn, Dipolar Bose-Einstein condensates with dipole-dependent scattering length, Phys. Rev. A 74 (2006), 033611.

17. L. Santos, G. V. Shlyapnikov, P. Zoller, and M. Lewenstein, Bose-Einstein condensation in trapped dipolar gases, Phys. Rev. Lett. 85 (2000), no. 9, 1791-1794.

18. E. M. Stein, Singular integrals and differentiability properties of functions, Princeton Mathematical Series, No. 30, Princeton University Press, Princeton, N.J., 1970.

19. Y. Tsutsumi, $L^{2}$-solutions for nonlinear Schrödinger equations and nonlinear groups, Funkcial. Ekvac. 30 (1987), no. 1, 115-125.

20. K. Yajima, Existence of solutions for Schrödinger evolution equations, Comm. Math. Phys. 110 (1987), 415-426.

21. S. Yi and L. You, Trapped atomic condensates with anisotropic interactions, Phys. Rev. A 61 (2000), no. 4, 041604

22. - Trapped condensates of atoms with dipole interactions, Phys. Rev. A 63 (2001), no. 5, 053607.

23. _ Calibrating dipolar interaction in an atomic condensate, Phys. Rev. Lett. 92 (2004), no. 19, 193201.

24. V. E. Zakharov, Collapse of Langmuir waves, Sov. Phys. JETP 35 (1972), 908-914.

(R. Carles) CNRS \& Université Montpellier 2, Mathématiques, CC 051, Place Eugène Bataillon, 34095 Montpellier Cedex 5, France

E-mail address: Remi.Carles@math.cnrs.fr

(P. Markowich) Department of Applied Mathematics and Theoretical Physics, CMS, Wilberforce Road, Cambridge CB3 0WA, England

E-mail address: p.a.markowich@damtp.cam.ac.uk

(C. Sparber) Department of Applied Mathematics and Theoretical Physics, CMS, Wilberforce Road, Cambridge CB3 0WA, England

E-mail address: c.sparber@damtp.cam.ac.uk 